\title{
GENERALIZED QUOTIENTS IN COXETER GROUPS
}

\author{
ANDERS BJÖRNER AND MICHELLE L. WACHS
}

Abstract. For $(W, S)$ a Coxeter group, we study sets of the form

$$
W / V=\{w \in W \mid l(w v)=l(w)+l(v) \text { for all } v \in V\},
$$

where $V \subseteq W$. Such sets $W / V$, here called generalized quotients, are shown to have much of the rich combinatorial structure under Bruhat order that has previously been known only for the case when $V \subseteq S$ (i.e., for minimal coset representatives modulo a parabolic subgroup). We show that Bruhat intervals in $W / V$, for general $V \subseteq W$, are lexicographically shellable. The Möbius function on $W / V$ under Bruhat order takes values in $\{-1,0,+1\}$.

For finite groups $W$, generalized quotients are the same thing as lower intervals in the weak order. This is, however, in general not true. Connections with the weak order are explored and it is shown that $W / V$ is always a complete meet-semilattice and a convex order ideal as a subset of $W$ under weak order.

Descent classes $D_{I}=\{w \in W \mid l(w s)<l(w) \Leftrightarrow s \in I$, for all $s \in S\}$, $I \subseteq S$, are also analyzed using generalized quotients. It is shown that each descent class, as a poset under Bruhat order or weak order, is isomorphic to a generalized quotient under the corresponding ordering.

The latter half of the paper is devoted to the symmetric group and to the study of some specific examples of generalized quotients which arise in combinatorics. For instance, the set of standard Young tableaux of a fixed shape or the set of linear extensions of a rooted forest, suitably interpreted, form generalized quotients. We prove a factorization result for the quotients that come from rooted forests, which shows that algebraically these quotients behave as a system of minimal "coset" representatives of a subset which is in general not a subgroup. We also study the rank generating function for certain quotients in the symmetric group.

1. Introduction. This paper is concerned with the combinatorial study of Coxeter groups under two well-known partial orderings, Bruhat order and weak order. We introduce and study a class of subsets of Coxeter groups, which as ordered sets exhibit many of the same structural properties as the systems of minimal length coset representatives modulo parabolic subgroups.

Some familiarity with Coxeter groups will be assumed, such as what can be found in the first few pages of [12]. The basic properties of Bruhat and weak order will be reviewed in the next section.

Throughout this paper $(W, S)$ will denote a Coxeter group $W$ with distinguished generating set $S$. The length $l(w)$ of $w \in W$ is the least $q$ such that $w=s_{1} s_{2} \cdots s_{q}$,

Received by the editors May 28, 1987. Presented to the American Mathematical Society at the annual meeting in Anaheim, California, January 1985.

1980 Mathematics Subject Classification (1985 Revision). Primary 05A99; Secondary 06F99, 20B30, $20 \mathrm{~F} 99$.

Both authors acknowledge partial support from the National Science Foundation. 
with $s_{i} \in S$. Such a minimum length expression is called a reduced expression for $w$. Subgroups $W_{J}$ generated by subsets $J$ of $S$ are called parabolic subgroups.

For $J \subseteq S$, let

$$
W^{J}=\{w \in W \mid l(w s)=l(w)+1 \text { for all } s \in J\} .
$$

We will call such subsets $W^{J}$ ordinary quotients of $W$. One basic fact about $W^{J}$ (cf. [12, p. 37]) is that every $w \in W$ can be uniquely factorized $w=u \cdot v$, so that $u \in W^{J}$ and $v \in W_{J}$. Furthermore, this factorization satisfies $l(w)=l(u)+l(v)$. In particular, $W^{J}$ intersects each left coset of $W_{J}$ in its unique element of minimum length.

We now generalize this concept as follows. For $V \subseteq W$, let

$$
W / V=\{w \in W \mid l(w v)=l(w)+l(v) \text { for all } v \in V\} .
$$

The subsets of $W$ of the form $W / V$ will be called generalized quotients. The name is motivated by the fact that for $J \subseteq S$ we have: $W / J=W^{J}=W / W_{J}$. It will be shown that generalized quotients have many structural properties in common with ordinary quotients, and that they are helpful for the study of some other classes of subsets in Coxeter groups, such as descent classes and classes of tableau words and linear extensions of posets in the symmetric group.

The paper is organized as follows.

In $\S 2$ we review some basic facts about Bruhat order and weak order on Coxeter groups.

$\S 3$ is devoted to the study of generalized quotients $W / V$ as ordered sets under Bruhat order. It is shown that $W / V$ has the following chain property: if $u<w$ in $W / V$ then there exists a chain $u=u_{0}<u_{1}<\cdots<u_{k}=w$ in $W / V$ such that $l\left(u_{i}\right)=l(u)+i$, for $1 \leq i \leq k$. Also, if $W / V$ is finite then it has a unique maximal element. The main result of $\S 3$ is that every Bruhat interval $[u, w]$ in $W / V$ is lexicographically shellable (cf. Definition 3.1). From this combinatorial property we deduce that the simplicial complex of chains in a nonempty open Bruhat interval $(u, w)$ of $W / V$ triangulates a sphere or a ball, and is therefore Cohen-Macaulay. This was previously shown for ordinary quotients by the authors in [8]. It also follows that the Möbius function $\mu(u, w)$ on $W / V$ takes values in $\{-1,0,+1\}$, extending a result of Verma [23] and Deodhar [13] for ordinary quotients.

In $\S 4$ we explore relationships between generalized quotients and intervals in the left weak ordering of the full Coxeter group. We show that for finite Coxeter groups the following three classes of subsets coincide: (i) generalized quotients $W / V$ for arbitrary $V \subseteq W$, (ii) generalized quotients $W /\{v\}$ for singleton $\{v\} \subseteq W$, (iii) lower left intervals $[e, u]_{L}$. For infinite $W$, and especially for infinite $W / V$, the situation becomes more complicated; there are lower left intervals which are not generalized quotients (and, of course, conversely) and there are generalized "ivotients which cannot be realized by a singleton $V$. We show that as an ordered set under left weak order, $W / V$ is a complete meet-semilattice, and if finite is a lattice. For the full group $W$ this had earlier been shown by Björner $[\mathbf{5}, \mathbf{7}]$.

Let $T=\left\{w s w^{-1} \mid s \in S, w \in W\right\}$, and for $A \subseteq T$ define

$$
W^{A}=\{w \in W \mid l(w t)>l(w) \text { for all } t \in A\} .
$$


Since $S \subseteq T$, this extends the definition (1.1) of an ordinary quotient in a different way than (1.2). In $\S 5$ we consider such "alternative" generalized quotients $W^{A}$. It is shown that every generalized quotient $W / V$ and every lower left interval $[e, u]_{L}$ is of the form $W^{A}$, for some $A \subseteq T$. We leave open the question of whether some of the properties we prove for $W / V$ extend to the more general $W^{A}$. In an appendix (§10) we discuss a notion of convexity and a theorem of J. Tits [22], which in our language says that a subset of $W$ is of the form $W^{A}$ if and only if it is a convex order ideal in the left weak ordering of $W$.

For $I \subseteq J \subseteq S$, define the descent class

$$
D_{I}^{J}=\{w \in W \mid s \in I \Rightarrow l(w s)<l(w) \text { and } s \in S-J \Rightarrow l(w s)>l(w)\} .
$$

Again, the ordinary quotients are special cases: $W^{J}=D_{\varnothing}^{S-J}$. We show in $\S 6$ that every descent class $D_{I}^{J}$ is isomorphic to a generalized quotient under both Bruhat order and left weak order. Hence, the structural properties shown in $\S \S 3$ and 4 hold also for descent classes, as ordered sets. We show that descent classes $D_{I}^{I}$ break up further into smaller pieces, each isomorphic to a generalized quotient. Such partitionings are induced by $J$, for all $I \subseteq J \subseteq S$. Consequently, the Bruhat ordering of $W$ can be nontrivially partitioned into disjoint lexicographically shellable subposets in a multitude of ways. We study mapping and embedding properties among the blocks of such partitions.

The last 3 sections are devoted to the symmetric group, and in particular to the study of some specific generalized quotients in $\mathscr{S}_{n}$ which arise by combinatorial constructions. First, let $\lambda / \mu$ be a skew shape and consider the set $\mathscr{T}_{\lambda / \mu}$ of all standard Young tableaux of shape $\lambda / \mu$. Reading the tableaux row by row (other reading orders are also possible) we get a set $w\left(\mathscr{T}_{\lambda / \mu}\right)$ of permutations, the "tableau words". We show that $w\left(\mathscr{T}_{\lambda / \mu}\right)$ is a generalized quotient. Second, let $\varphi$ be a planar forest and consider the set $\mathscr{F}_{\varphi}$ of all standard labelings of $\varphi$ (i.e., bijective labelings of the nodes $x_{1}, x_{2}, \ldots, x_{n}$ of $\varphi$ with the integers $1,2, \ldots, n$, such that the label of any node is greater than those of all its children). Reading the standard labeling in postorder (other reading orders are also possible) we get a set $w\left(\mathscr{F}_{\varphi}\right)$ of permutations, which again forms a generalized quotient. We call sets of the forms $w\left(\mathscr{T}_{\lambda / \mu}\right)$ and $w\left(\mathscr{F}_{\varphi}\right)$, tableau quotients and forest quotients, respectively.

The permutations in an ordinary quotient $W^{J}$ in the symmetric group can be characterized either as being piecewise monotone (i.e., having ascents in certain specified positions), or else as being permutations of multisets or "shuffles". A descent class $D_{I}^{J}$ in $\mathscr{S}_{n}$ consists of those permutations having descents in the positions specified by $I$ and ascents in the positions specified by $S-J$. As a tableau quotient, $W^{J}$ corresponds to a shape with nonoverlapping rows, and $D_{I}^{J}$ to a shape with rows which overlap in at most one box (for "French" reading order, cf. §7). As a forest quotient, $W^{J}$ corresponds to a forest consisting only of linear trees.

Tableau quotients and forest quotients are studied in $\S \S 7$ and 8 , respectively. A property of forest quotients $w\left(\mathscr{F}_{\varphi}\right)$, which is particularly interesting, is that they split the symmetric group. By this we mean that given a planar forest $\varphi$ on $n$ nodes there exists a subset $V_{\varphi} \subseteq \mathscr{S}_{n}$ (actually, $V_{\varphi}$ is an interval in right weak order), such that every $w \in \mathscr{S}_{n}$ has a unique factorization $w=u v$, where $u \in w\left(\mathscr{F}_{\varphi}\right)$ and $v \in V_{\varphi}$. Furthermore, then also $l(w)=l(u)+l(v)$. Hence, forest quotients behave 
algebraically very much like ordinary quotients. They act as a system of minimal "coset" representatives of a subset which is in general not a subgroup.

For $A \subseteq \mathscr{S}_{n}$, let $A(q)=\sum q^{l(w)}$, with summation over all $w \in A$. A well-known result, due to MacMahon $\left[\mathbf{1}\right.$, p. 41], is that for ordinary quotients $W^{J}(q)$ equals a $q$-multinomial coefficient. In $\S 9$ we study the polynomials $A(q)$ for generalized quotients in the symmetric group. For forest quotients $A=w\left(\mathscr{F}_{\varphi}\right)$, we show that $A$ is rank symmetric and provide an explicit formula for $A(q)$, which is a $q$-analogue of Knuth's hook-length formula for planar forests. For forests with linear trees (i.e., ordinary quotients), it reduces to MacMahon's $q$-multinomial formula. For descent classes $A=D_{I}^{J}$, we establish a determinantal formula for $A(q)$, which for the case $I=\varnothing$ specializes to the $q$-multinomial coefficient, for $q=1$ and $I=J$ to another result of MacMahon, and for $I=J$ to a formula of Stanley. Finally, we show that certain left intervals in $\mathscr{S}_{n}$ related to forest quotients have the symmetric chain decomposition property as posets under Bruhat order. This extends the known fact that the parabolic subgroups (or Young subgroups, as they are often called for $\mathscr{S}_{n}$ ) have symmetric chain decompositions.

2. Background on the partial orderings of Coxeter groups. We begin by reviewing the definitions of Bruhat order and weak order. Let $(W, S)$ be a Coxeter group, and let $T$ be the set of conjugates of $S$, i.e., $T=\left\{w s w^{-1} \mid w \in W, s \in S\right\}$. For $u, w \in W$ we say that $u$ precedes $w$ in Bruhat order, written $u<_{B} w$, if there exist $t_{1}, t_{2}, \ldots, t_{m} \in T$ such that $l\left(t_{i} t_{i-1} \cdots t_{1} u\right)>l\left(t_{i-1} \cdots t_{1} u\right)$ for $i=1,2, \ldots, m$ and $t_{m} t_{m-1} \cdots t_{1} u=w$. Similarly we say $u$ precedes $w$ in left order, written $u<_{L} w$, if there exist $s_{1}, s_{2}, \ldots, s_{m} \in S$ such that $l\left(s_{i} s_{i-1} \cdots s_{1} u\right)=l(u)+i$ for $i=1,2, \ldots, m$ and $s_{m} s_{m-1} \cdots s_{1} u=w$. If in the definition of left order we place the generators $s_{i}$ to the right of $u$ rather than to the left we define a partial order relation that we call right order and denote by $<_{R}$.

It is obvious that $u<_{L} w$ implies $u<_{B} w$. It is also true that $u<_{R} w$ implies $u<_{B} w$, since $u s=u s u^{-1} \cdot u$. Left order and right order are clearly isomorphic partial orderings. They are sometimes referred to in the literature as weak (Bruhat) order; and Bruhat order is also known as strong order. We will use the term weak order when there is no need to distinguish between left and right order. For an expository discussion of Bruhat order and weak order on Coxeter groups see [5].

For $u, v \in W$ and $u \leq_{L} v$ the set $\left\{w \in W \mid u \leq_{L} w \leq_{L} v\right\}$ will be called a left interval and will be denoted by $[u, v]_{L}$. A lower left interval is a left interval of the form $[e, v]_{L}$ where $e$ is the identity element of $W$. We define Bruhat interval $[u, v]_{B}$ and right interval $[u, v]_{R}$ similarly. Other poset constructs will also be preceded by the terms "left", "right", or "Bruhat", to distinguish between the respective partial orderings. For example, a left maximal element of a subset $U$ of $W$ is an element of $U$ that is maximal in the left order on $U$.

We now review some well-known properties of Bruhat order which are important tools in this paper. Proofs can be found e.g. in [13]. For each $w \in W$, let $T_{w}=$ $\left\{t \in T \mid t w<_{B} w\right\}$.

Strong EXChANGE PROPERTy. For $w \in W$ and $w=s_{1} s_{2} \cdots s_{k}, s_{i} \in S$, if $t \in T_{w}$ then $t w=s_{1} s_{2} \cdots \widehat{s}_{i} \cdots s_{k}$ ( $s_{i}$ deleted) for some $i=1,2, \ldots, k$. Furthermore, if $s_{1} s_{2} \cdots s_{k}$ is a reduced expression then $i$ is uniquely determined and $T_{w}=\left\{s_{1} s_{2} \cdots s_{i} s_{i-1} \cdots s_{1} \mid i=1,2, \ldots, k\right\}$. 
A consequence of the strong exchange property is the following characterization of Bruhat order.

SUBWORD PROPERTY. Let $v=s_{1} s_{2} \cdots s_{q}$ be a reduced expression. Then $u \leq_{B} v$ if and only if there is a reduced expression for $u$ which is a subword of $s_{1} s_{2} \cdots s_{q}$, i.e., $u=s_{i_{1}} s_{i_{2}} \cdots s_{i_{j}}$ where $1 \leq i_{1}<i_{2}<\cdots<i_{j} \leq q$.

The following is a corollary of the subword property.

LIFTING PROPERTY. If $s u>_{L} u$ and $s v>_{L} v$, then the following are equivalent:

(i) $v \geq_{B} u$,

(ii) $s v>_{B} u$,

(iii) $s v \geq_{B} s u$.

It is well known that the weak orders and Bruhat order have the following basic combinatorial properties:

(1) The identity $e$ is the minimum element in all three orders.

(2) Under all three orders $W$ is ranked, and the poset rank function is the same as the group-theoretic length function $l$. (By a ranked poset we mean a poset $P$ such that for each $x \in P$ all maximal chains in $\{y \in P \mid y \leq x\}$ have the same finite length, called the rank of $x$.)

(3) When $W$ is finite, $W$ has an element $w_{0}$ which is maximum in all three orders.

Recall that a graded poset is a ranked poset with a minimum and a maximum element (usually denoted $\widehat{0}$ and $\widehat{1}$ ). Hence a finite Coxeter group $W$ is graded as a poset under all three orderings. For a finite parabolic subgroup $W_{J}$, we shall denote its maximum element by $w_{0}(J)$. This maximum is characterized by the following well-known property, cf. [12, p. 43]. (The nontrivial part, the "if" direction, happens to also be a consequence of Theorem 4.1 below.)

Proposition 2.1. Let $J \subseteq S$ and $v \in W_{J}$. Then $W_{J}$ is finite and $v=w_{0}(J)$ if and only if $s v<_{L} v$ for all $s \in J$.

The following lemmas and propositions will also be needed in the sections that follow.

LEMMA 2.2. Let $a, b, u \in W$ and $l(a u)=l(a)+l(u)$ and $l(b u)=l(b)+l(u)$. Then $a u<_{B}$ bu if and only if $a<_{B} b$.

ProOF. The fact that $a<_{B} b$ implies $a u<_{B} b u$ follows from the subword property. We shall use induction on $l(u)^{\prime}$ to prove the converse. For $l(u)=0$ the result is trivial. Assume $l(u)>0$ and $a u<_{B} b u$. Then $u=u^{\prime} s$ for some $s \in S$, where $l\left(u^{\prime}\right)=l(u)-1$. Since $a u^{\prime}<_{R} a u$ and $b u^{\prime}<_{R} b u$, the lifting property implies that $a u^{\prime}<_{B} b u^{\prime}$. It follows by induction that $a<_{B} b$.

Note that Lemma 2.2 holds trivially for the left order. An immediate consequence of Lemma 2.2 is the following proposition.

Proposition 2.3. Let $u, v \in W$ and $u<_{L} v$. Then the map $\varphi:[u, v]_{L} \rightarrow$ $\left[e, v u^{-1}\right]_{L}$ defined by $\varphi(w)=w u^{-1}$ is an isomorphism under Bruhat order and left order.

Proposition 2.3 shows that any analysis of left order or Bruhat order on left intervals can be restricted to lower left intervals. 
We shall make use of the following notation: For $w \in W$ let $\langle w\rangle$ denote any reduced expression for $w$. Let $\langle u\rangle\langle v\rangle$ denote the concatenation of reduced expressions for $u$ and $v$. Saying that $\langle u\rangle\langle v\rangle$ is reduced is a convenient way of saying that $l(u v)=l(u)+l(v)$.

LEMMA 2.4. Let $u, v \in W$. Suppose for some $t \in T$ that $t u>_{B} u$ but tuv $<_{B}$ $u v$. Then there exists $\widehat{v}<_{B} v$ such that tuv $=u \widehat{v}$. Furthermore, if $\langle u\rangle\langle v\rangle$ is reduced and $t \in S$ then $l(\widehat{v})=l(v)-1$ and $\langle u\rangle\langle\widehat{v}\rangle$ is reduced.

PROOF. By the strong exchange and subword properties either $t u v=\widehat{u} v$ or $t u v=u \widehat{v}$, where $\widehat{u}<_{B} u$ or $\widehat{v}<_{B} v$, respectively. The first case would produce the contradiction $t u=\widehat{u}<_{B} u$. Hence, $t u v=u \widehat{v}$. Now, if $\langle u\rangle\langle v\rangle$ is reduced and $t \in S$ then

$$
l(u)+l(v)>l(u)+l(\widehat{v}) \geq l(u \widehat{v})=l(t u v)=l(u v)-1=l(u)+l(v)-1,
$$

from which the rest follows.

The following characterization of the right ordering is known from [5]. We include a proof here for completeness.

Proposition 2.5. For all $u, w \in W, u \leq_{R} w$ if and only if $T_{u} \subseteq T_{w}$.

Proof. $(\Rightarrow)$ Choose any reduced expression $s_{1} s_{2} \cdots s_{k}$ for $u$. Since $u \leq_{R} w$, there is a reduced expression of the form $s_{1} s_{2} \cdots s_{k} s_{k+1} \cdots s_{j}$ for $w$. By the strong exchange property

$$
\begin{aligned}
T_{u} & =\left\{s_{1} s_{2} \cdots s_{i} s_{i-1} \cdots s_{1} \mid i=1,2, \ldots, k\right\} \\
& \subseteq\left\{s_{1} s_{2} \cdots s_{i} s_{i-1} \cdots s_{1} \mid i=1,2, \ldots, j\right\}=T_{w} .
\end{aligned}
$$

$(\Leftarrow)$ Let $u=s_{1} s_{2} \cdots s_{k}$ be a reduced expression and let $t_{i}=s_{1} s_{2} \cdots s_{i} s_{i-1} \cdots s_{1}$ for $i=1,2, \ldots, k$. Then by the strong exchange property, $T_{u}=\left\{t_{1}, t_{2}, \ldots, t_{k}\right\}$ and the $t_{i}$ are distinct. Hence, $t_{i} \in T_{w}$ for all $i=1,2, \ldots, k$. We shall prove, by induction on $i$, that $s_{1} s_{2} \cdots s_{i} \leq_{R} w$ holds for all $i=0,1,2, \ldots, k$. Clearly, the assertion is true for $i=0$. Suppose it is true for $i-1$ where $1 \leq i \leq k$. This means that $s_{1} s_{2} \cdots s_{i-1} v=w$ where $s_{1} s_{2} \cdots s_{i-1}\langle v\rangle$ is reduced. Clearly, $t_{i} s_{1} s_{2} \cdots s_{i-1}=s_{1} s_{2} \cdots s_{i}>_{B} s_{1} s_{2} \cdots s_{i-1}$. Since we also have $t_{i} w<_{B} w$, Lemma 2.4 implies that $t_{i} w=s_{1} s_{2} \cdots s_{i-1} \widehat{v}$ where $\widehat{v}<_{B} v$. It follows that

$$
w=t_{i} t_{i} w=t_{i} s_{1} s_{2} \cdots s_{i-1} \widehat{v}=s_{1} s_{2} \cdots s_{i} \widehat{v} .
$$

We have

$$
l(\widehat{v})+i \geq l(w)=l(v)+i-1 \geq l(\widehat{v})+i,
$$

from which it follows that $s_{1} s_{2} \cdots s_{i}\langle\widehat{v}\rangle$ is reduced. Hence, $s_{1} s_{2} \cdots s_{i} \leq_{R} w$. We may now conclude that $s_{1} s_{2} \cdots s_{k} \leq_{R} w$, as desired.

It is an elementary observation that combinatorial facts about reduced decompositions, partial orderings, etc., in a Coxeter group can be mirrored into corresponding "dual" facts by systematically reversing the ordering of expressions and interchanging left and right. For example, all properties, lemmas and propositions mentioned in this section have such dual counterparts (except that the subword property is self-dual). Formally, this duality can be achieved by passing to inverse elements. We shall use this repeatedly without further special mention. 
3. Bruhat order on generalized quotients. In this section some properties of Bruhat order on full Coxeter groups are extended to generalized quotients, $W / V$. It is an immediate consequence of the definition that $e$ is the minimum element of $W / V$. The fact that $W / V$ is ranked under Bruhat order is, however, not immediate. We will prove this and establish lexicographic shellability at the same time. Lexicographic shellability was shown for all ordinary quotients by the authors in [8].

Let $P$ be a graded poset of length $r$, i.e., the rank of the maximal element $\widehat{1}$ is $r$. The symbol $\rightarrow$ shall be used to denote the covering relation in $P$, i.e., $a \rightarrow b$ means that $a$ covers $b$ in $P$. To each maximal chain $m: \hat{1}=x_{0} \rightarrow x_{1} \rightarrow \cdots \rightarrow x_{r}=\widehat{0}$ of $P$, we assign a label sequence $\lambda(m)=\left(\lambda_{1}(m), \lambda_{2}(m), \ldots, \lambda_{r}(m)\right) \in Z^{r}$ satisfying the following condition: If two maximal chains $m$ and $m^{\prime}$ coincide along their first $k$ edges (i.e., top $k$ edges in the Hasse diagram) then $\lambda_{i}(m)=\lambda_{i}\left(m^{\prime}\right)$ for $i=1,2, \ldots, k$. If $[x, y]$ is an interval and $c$ is an unrefinable chain from $y$ to $\hat{1}$, the pair $([x, y], c)$ will be called a rooted interval. A well-defined labeling $\lambda^{c}$ of the maximal chains of $[x, y]$ is induced by the chain $c$. Indeed, if $b$ is a maximal chain of $[x, y]$ then $\lambda_{i}^{c}(b)=\lambda_{r-r(y)+i}(m)$ for $i=1,2, \ldots, r(y)-r(x)$, where $m$ is any maximal chain of $P$ which contains $c$ and $b$, and $r(x)$ and $r(y)$ are the respective ranks of $x$ and $y$.

DEFINITION 3.1. A labeling $\lambda$ of maximal chains of a graded poset $P$ as above is a chain lexicographic labeling $(C L$-labeling $)$ if for every rooted interval $([x, y], c)$ in $P$,

(i) there is a unique maximal chain $a$ in $[x, y]$ which is increasing, i.e., $\lambda_{1}^{c}(a) \leq$ $\lambda_{2}^{c}(a) \leq \cdots \leq \lambda_{r(y)-r(x)}^{c}(a)$, and

(ii) if $b$ is any other maximal chain in $[x, y]$ then the label sequence $\lambda^{c}(a)$ is lexicographically smaller than the label sequence $\lambda^{c}(b)$.

The poset $P$ is said to be chain lexicographically shellable $(C L$-shellable) if it admits a CL-labeling. See $[\mathbf{4}, \mathbf{8}, \mathbf{9}]$ for further details concerning this concept.

We now describe the CL-labeling of Bruhat intervals of Coxeter groups that appears in [8]. Let $u<_{B} w$ and let $s_{1} s_{2} \cdots s_{q}$ be a reduced expression for $w$. Suppose that $l(w)-l(u)=r$ and let $m: w \rightarrow w_{1} \rightarrow w_{2} \rightarrow \cdots \rightarrow w_{r}=u$ be a Bruhat maximal chain of $[u, w]_{B}$. We assign label sequence $\lambda(m)=\left(\lambda_{1}(m), \lambda_{2}(m), \ldots, \lambda_{r}(m)\right)$ to $m$ as follows: By the strong exchange property $w_{1}=t_{1} w=s_{1} s_{2} \cdots \widehat{s}_{i} \cdots s_{q}$ where the deleted generator $s_{i}$ is uniquely determined. Let $\lambda_{1}(m)=i$. Now repeat the process. After $k$ deletions we have reached $w_{k}$ and obtained a uniquely determined subword expression $w_{k}=s_{j_{1}} s_{j_{2}} \cdots s_{j_{q-k}}, 1 \leq j_{1}<j_{2}<\cdots<j_{q-k} \leq q$. Again we have $w_{k+1}=t_{k+1} w_{k}=s_{j_{1}} s_{j_{2}} \cdots \hat{s}_{j_{i}} \cdots s_{j_{q-k}}$ where the deleted generator is uniquely determined. Let $\lambda_{k+1}(m)=j_{i}$. Hence the idea is to label by the positions of the generators which are successively deleted from the chosen reduced expression for $w$ as we go down the maximal chain from $w$ to $u$. We shall call this labeling the subword labeling of $[u, w]_{B}$ induced by the reduced expression for $w$. Note that different reduced expressions for $w$ in general induce different subword labelings of $[u, w]_{B}$.

EXAMPLE. The subword labeling of the symmetric group $\mathscr{S}_{3}$ induced by the reduced expression $a b a$, where $a$ and $b$ are the adjacent transpositions $(1,2)$ and 
$(2,3)$, respectively, is given by

$$
\begin{aligned}
& \lambda(a b a \rightarrow b a \rightarrow a \rightarrow e)=(1,2,3), \\
& \lambda(a b a \rightarrow b a \rightarrow b \rightarrow e)=(1,3,2), \\
& \lambda(a b a \rightarrow a b \rightarrow a \rightarrow e)=(3,2,1), \\
& \lambda(a b a \rightarrow a b \rightarrow b \rightarrow e)=(3,1,2) .
\end{aligned}
$$

THEOREM 3.2 [8]. For any Coxeter group $W$, if $u<_{B} w$ then the subword labeling of $[u, w]_{B}$ induced by any reduced expression for $w$ is a CL-labeling.

To extend this result to generalized quotients we make use of the following lemma, cf. [4, Proposition 2.8].

LemMA 3.3. Suppose that $P$ is $C L$-shellable with CL-labeling $\lambda$. Suppose that $Q$ is a subposet of $P$ such that

(i) $\widehat{0}, \widehat{1} \in Q$, and

(ii) for every rooted interval $([x, y], c)$ of $P$ such that $x, y \in Q$ and $c \subseteq Q$, the unique increasing maximal chain of $[x, y]$ lies entirely in $Q$.

Then $Q$ under the inherited order is a CL-shellable poset of equal rank, with $C L$ labeling the restriction of $\lambda$.

PROOF. The verification is straightforward.

THEOREM 3.4. Let $W / V$ be a generalized quotient, $V \subseteq W$. Suppose $u, w \in$ $W / V$ and $u<_{B} w$. Then for the subword labeling of $[u, w]_{B}$ induced by any reduced expression for $w$, the unique increasing maximal chain of $[u, w]_{B}$ lies entirely in $W / V$.

PROOF. Fix a reduced expression $s_{1} s_{2} \cdots s_{q}$ for $w$. Let $m$ be the unique increasing maximal chain of $[u, w]_{B}$ in the subword labeling induced by $s_{1} s_{2} \cdots s_{q}$. We shall show by induction on $l(z)$ that $z \in W / V$ for all $z \in m$. If $l(z)=l(u)$ then $z=u$ and hence $z \in W / V$. Suppose now that $l(z)>l(u)$ and $z^{\prime} \in W / V$ for all $z^{\prime} \in m$ such that $l\left(z^{\prime}\right)<l(z)$. Assume that $z \notin W / V$. Then there is some $v \in V$ such that $\langle z\rangle\langle v\rangle$ is not reduced. Let $v=v_{1} s v_{2}$ where $\left\langle v_{1}\right\rangle s\left\langle v_{2}\right\rangle$ and $\langle z\rangle\left\langle v_{1}\right\rangle$ are reduced and $\langle z\rangle\left\langle v_{1}\right\rangle s$ is not reduced. It follows that $z v_{1} s<_{R} z v_{1}$.

Let $\widehat{z}$ be the element of $m$ which is covered by $z$. Then by the induction hypothesis, $\langle\widehat{z}\rangle\langle v\rangle$ is reduced which implies that $\langle\widehat{z}\rangle\left\langle v_{1}\right\rangle s$ is also reduced. We have $\widehat{z} v_{1} s>_{R} \widehat{z} v_{1}$ and $z v_{1} s<_{R} z v_{1}$. Since $\widehat{z}<_{B} z$, the subword property implies that $\widehat{z} v_{1}<_{B} z v_{1}$. By the lifting property we have $\widehat{z} v_{1} \leq_{B} z v_{1} s$. Since $l\left(\widehat{z} v_{1}\right)=l\left(z v_{1}\right)-1$, we conclude that $\widehat{z} v_{1}=z v_{1} s$.

Since $m$ has increasing labels, it follows that

$$
z v_{1}=s_{1} \cdots \widehat{s}_{i_{1}} \cdots \widehat{s}_{i_{2}} \cdots \widehat{s}_{i_{k-1}} \cdots s_{q} v_{1}
$$

and

$$
\widehat{z} v_{1}=s_{1} \cdots \widehat{s}_{i_{1}} \cdots \widehat{s}_{i_{2}} \cdots \widehat{s}_{i_{k-1}} \cdots \widehat{s}_{i_{k}} \cdots s_{q} v_{1}
$$

where $k-1=l(w)-l(z)$. Since $\widehat{z} v_{1}=z v_{1} s$, we can conclude that

$$
s=v_{1}^{-1} s_{q} s_{q-1} \cdots s_{i_{k}} s_{i_{k}+1} \cdots s_{q} v_{1}
$$

Consequently, $w v_{1} s=s_{1} s_{2} \cdots \widehat{s}_{i_{k}} \cdots s_{q} v_{1}$. This implies that $\langle w\rangle\left\langle v_{1}\right\rangle s$ is not reduced which in turn implies that $\langle w\rangle\langle v\rangle$ is not reduced. Since this contradicts the fact that $w \in W / V$, we are done. 
COROLlARY 3.5. Bruhat order on $W / V$ has the chain property.

The chain property (as defined in $\S 1$ ) directly implies that $W / V$ is a ranked poset whose order-theoretic rank function coincides with the group-theoretic length function. For ordinary quotients this was previously shown by Deodhar [13, Corollary 3.8] using a completely different argument.

COROLlARY 3.6. Every Bruhat interval of $W / V$ is $C L$-shellable under Bruhat order.

ProOF. Bruhat intervals of $W / V$ have the form $[a, b]_{B} \cap W / V$, where $a, b \in W / V$ and $a<_{B} b$. Let $P$ be the poset $[a, b]_{B}$ under Bruhat order and let $Q$ be the subposet $[a, b]_{B} \cap W / V$. We shall use Lemma 3.3 to conclude that $Q$ is CL-shellable.

Clearly $Q$ satisfies (i) of Lemma 3.3. Note (as in [8]) that any labeling of a rooted interval, $\left([u, w]_{B}, c\right)$, of $P$ induced by a subword labeling of $P$ is itself a subword labeling of $[u, w]_{B}$. Therefore by Theorem 3.4, if $u, w \in W / V$ then the unique increasing maximal chain of $[u, w]_{B}$ for this labeling lies in $W / V$. It follows that $Q$ satisfies (ii) of Lemma 3.3. Hence by Lemma 3.3, $Q$ is CL-shellable.

Some notable consequences of the shellability of ordinary quotients are discussed in [8]. These results now hold for generalized quotients as well and their proofs go through exactly as in [8]. We shall merely state some of these results here:

(1) Let $\mu$ be the Möbius function for Bruhat order on $W / V$.

If $u, w \in W / V$ and $u \leq_{B} w$ then

$$
\mu(u, w)= \begin{cases}(-1)^{l(w)-l(u)}, & \text { if }[u, w]_{B} \subseteq W / V \\ 0, & \text { otherwise. }\end{cases}
$$

(2) Let $u, w \in W / V, u<_{B} w$, and $l(w)-l(u)=d+2 \geq 2$. Then the simplicial complex of Bruhat chains of the open interval $(u, w)_{B} \cap W / V$ is a triangulation of a $d$-sphere if $[u, w]_{B} \subseteq W / V$ and is a triangulation of a $d$-cell otherwise.

(3) The Stanley-Reisner ring of the simplicial complex in (2) is Cohen-Macaulay; and it is Gorenstein if $[u, w]_{B} \subseteq W / V$.

Having established that generalized quotients are ranked under Bruhat order, our next goal is to show that finite generalized quotients have a maximum element. We shall, in fact, prove something slightly more general which holds for infinite as well as for finite generalized quotients.

THEOREM 3.7. Let $u, w \in W / V$ and let $z$ be a minimal upper bound of $u$ and $w$ in Bruhat order (i.e., $z \geq_{B} u, w$ and if $z \geq_{B} y \geq_{B} u$, w then $z=y$ ). Then $z \in W / V$.

ProOF. Suppose $z \notin W / V$. Then $\langle z\rangle\langle v\rangle$ is not reduced for some $v \in V$. Let $v=v_{1} s v_{2}$ where $\left\langle v_{1}\right\rangle s\left\langle v_{2}\right\rangle$ and $\langle z\rangle\left\langle v_{1}\right\rangle$ are reduced and $\langle z\rangle\left\langle v_{1}\right\rangle s$ is not reduced. Since $\left\langle v_{1}\right\rangle s$ is reduced, Lemma 2.4 implies that $z v_{1} s=\widehat{z} v_{1}$ where $\widehat{z}<_{B} z$ and $\langle\widehat{z}\rangle\left\langle v_{1}\right\rangle$ is reduced.

It follows from $u, w \in W / V$ that $\langle u\rangle\left\langle v_{1}\right\rangle$ and $\langle w\rangle\left\langle v_{1}\right\rangle$ are reduced and that $u v_{1} s>_{R} u v_{1}$ and $w v_{1} s>_{R} w v_{1}$. By Lemma 2.2, $z v_{1} \geq_{B} u v_{1}$ and $z v_{1} \geq_{B} w v_{1}$ : Hence by the lifting property, $\widehat{z} v_{1}=z v_{1} s \geq_{B} u v_{1}$ and $\widehat{z} v_{1}=z v_{1} s \geq_{B} w v_{1}$. It now follows from Lemma 2.2 that $\widehat{z} \geq_{B} u, w$. But this contradicts the minimality of $z$. Therefore $z \in W / V$.

A poset $P$ is said to be directed if every pair of elements has a common upper bound. 


\section{COROLlaRY 3.8. Bruhat order on $W / V$ is a directed poset.}

PROOF. This follows from Theorem 3.7 and the fact that $W$ is a directed poset under Bruhat order [9, Lemma 6.4]. It is also a consequence of Theorem 4.2.

COROLLARY 3.9. If $W / V$ is finite then $W / V$ has a maximum element under Bruhat order. Hence $W / V$ is a graded CL-shellable poset under Bruhat order.

An interesting property of Bruhat order on ordinary quotients and parabolic subgroups in finite $W$ is that they are isomorphic to their duals (see [21, p. 181]). This does not, however, hold for all generalized quotients. A finite parabolic subgroup $W_{J}$ satisfies the condition that its maximum element $w_{0}(J)$ is an involution, and an ordinary quotient $W^{J}$, in finite $W$, satisfies the condition that its maximum element $w_{0}^{J}=w_{0} w_{0}(J)$ is a product of $w_{0}$ and an involution. It turns out that either one of these conditions on the maximum element is all that is required to guarantee that a generalized quotient is isomorphic to its dual. Since in the next section we shall see that all finite generalized quotients are actually lower left intervals, we shall give the result for lower left intervals.

THEOREM 3.10. If $v \in W$ is an involution then $[e, v]_{L}$ is isomorphic to its dual under both Bruhat and left order. Moreover, if $W$ is finite then $\left[e, w_{0} v\right]_{L}$ and $\left[e, v w_{0}\right]_{L}$ are also isomorphic to their duals.

PROOF. Suppose that $x<_{L} v$, or equivalently, $l\left(v x^{-1}\right)+l(x)=l(v)$. Since $(x v)^{-1}=v x^{-1}$, we get $l\left(x^{-1}\right)+l(x v)=l(v)$. This means that the mapping $x \rightarrow x v$ permutes the interval $[e, v]_{L}$ so that $l(x v)=l(v)-l(x)$ for all $x \in[e, v]_{L}$. If $x, y \in[e, v]_{L}$ and $y=t x>_{B} x, t \in T$, it follows that $y v=t x v<_{B} x v$. Since Bruhat order is generated by such relations $x<_{B} t x$, we conclude that $x \leq_{B} y$ if and only if $x v \geq_{B} y v$ in $[e, v]_{L}$. The argument for left order is similar.

For any $x \in W$, let $\vec{\varphi}_{x}: W \rightarrow W$ be the map $\vec{\varphi}_{x}(w)=w x^{-1}$ and let $\overleftarrow{\varphi}_{x}: W \rightarrow W$ be the map $\bar{\varphi}_{x}(w)=x^{-1} w$. By Proposition 2.3 , for $x \leq_{L} y$, the restriction of $\vec{\varphi}_{x}$ is an isomorphism from $[x, y]_{L}$ to $\left[e, y x^{-1}\right]_{L}$ under Bruhat and left order. In the finite case, the maps $\vec{\varphi}_{w_{0}}$ and $\bar{\varphi}_{w_{0}}$ are well-known antiautomorphisms of $W$ under both orders (for $\vec{\varphi}_{w_{0}}$ this is a special case of the preceding paragraph). Hence, the restriction of the map $\vec{\varphi}_{v} \circ \overleftarrow{\varphi}_{w_{0}}$ is an antiautomorphism of $\left[e, w_{0} v\right]_{L}$, and the restriction of $\overleftarrow{\varphi}_{w_{0}} \circ \vec{\varphi}_{w_{0}}$ is an isomorphism from $\left[e, v w_{0}\right]_{L}$ to $\left[e, w_{0} v\right]_{L}$. This completes the proof.

4. Relationships with the weak order. It was shown by Björner $[5,7]$ that every Coxeter group under the weak order is a complete meet-semilattice and is a lattice if the group is finite. We shall extend this result to generalized quotients.

THEOREM 4.1. Suppose $W / V$ is a generalized quotient. If $W / V$ is infinite then $W / V$, under left order, is a complete meet-semilattice with no maximal elements. If $W / V$ is finite then $W / V$ is a lower left interval of $W$ and is therefore a lattice under left order.

To prove Theorem 4.1, we shall show that $W / V$, under left order, has a property similar to but weaker than that of being directed. A subset $U$ of $W$ is said to be cross directed if for every ordered pair $x, y \in U$ there is some $w \in U$ such that $x \leq_{B} w$ and $y \leq_{L} w$. 
THEOREM 4.2. All generalized quotients $W / V$ are cross directed. More precisely, if $x, y \in W / V$ then there is some $z \in W$ such that

(1) $z \leq_{B} x$,

(2) $z y \in W / V$,

(3) $x \leq_{B} z y$,

(4) $y \leq_{L} z y$.

PROOF. Let $s_{1} s_{2} \cdots s_{q}$ be a reduced expression for $x$. We will prove the following assertion by induction on $i$ :

ASSERTION. For any $y \in W / V$, if $s_{i+1} s_{i+2} \cdots s_{q} \leq_{B} y$, where $0 \leq i \leq q$, then there is some $z \in W$ such that

(1) $z \leq_{B} s_{1} s_{2} \cdots s_{i}$,

(2) $z y \in W / V$

(3) $x \leq_{B} z y$,

(4) $y \leq_{L} z y$.

PROOF OF ASSERTION. If $i=0$ then $z=e$ works. Suppose $i>0$ and $s_{i+1} s_{i+2} \cdots s_{q} \leq_{B} y$. If $s_{i} s_{i+1} \cdots s_{q} \leq_{B} y$ also, then by induction there is a $z^{\prime}$ satisfying (1) (with $i$ replaced by $i-1$ ), (2), (3), and (4). By choosing $z=z^{\prime}$, we have established the assertion.

Now suppose $s_{i} s_{i+1} \cdots s_{q} \$_{B} y$. Let $v$ be any element of $V$. It follows from Lemma 2.2 that $s_{i} s_{i+1} \cdots s_{q} v \$_{B} y v$ and $s_{i+1} s_{i+2} \cdots s_{q} v \leq_{B} y v$. If $s_{i} y v<_{L} y v$ then by the lifting property $s_{i} s_{i+1} \cdots s_{q} v \leq_{B} y v$. Since this is a contradiction, $s_{i} y v>_{L} y v$. Since $\langle y\rangle\langle v\rangle$ is reduced, $s_{i}\langle y\rangle\langle v\rangle$ is also reduced. It follows that $s_{i} y \in W / V$ since $v$ was an arbitrary element of $V$. We also have that $s_{i} y>_{L} y$. Hence the lifting property now implies that $s_{i} s_{i+1} \cdots s_{q} \leq_{B} s_{i} y$.

We are now able to apply the induction hypothesis to $s_{i} y$, since $s_{i} y \in W / V$ and $s_{i} s_{i+1} \cdots s_{q} \leq_{B} s_{i} y$. Let $z^{\prime}$ be such that

$\left(1^{\prime}\right) z^{\prime} \leq_{B} s_{1} s_{2} \cdots s_{i-1}$

$\left(2^{\prime}\right) z^{\prime} s_{i} y \in W / V$

$\left(3^{\prime}\right) x \leq_{B} z^{\prime} s_{i} y$,

$\left(4^{\prime}\right) s_{i} y \leq_{L} z^{\prime} s_{i} y$.

We conclude that $z=z^{\prime} s_{i}$ satisfies (1), (2), (3), and (4). Indeed, the lifting property implies that $z^{\prime} s_{i} \leq_{B} s_{1} s_{2} \cdots s_{i}$.

PROOF OF THEOREM 4.1. Clearly all generalized quotients are left order ideals of $W$ (i.e., $x \leq_{L} y \in W / V$ implies $\left.x \in W / V\right)$. Hence they inherit the complete meet-semilattice property from $W$. To complete the proof we need only show that any left-maximal element of $W / V$ is the left maximum element of $W / V$. It will follow from this that an infinite $W / V$ has no left maximal elements and a finite $W / V$ is a lower left interval.

Let $w$ be a left maximal element of $W / V$ and let $u$ be any element of $W / V$. Since $W / V$ is cross directed there are $z_{1}, z_{2} \in W / V$ such that $z_{1} \geq_{L} w, z_{1} \geq_{B} u$, $z_{2} \geq_{L} u$, and $z_{2} \geq_{B} w$. Since $w$ is left maximal in $W / V$, we have that $z_{1}=w$. Therefore $w \geq_{B} u$ for all $u \in W / V$. This means that $w$ is the Bruhat maximum element of $W / V$. It follows that $w=z_{2}$. Hence $w \geq_{L} u$ for all $u \in W / V$. We now have that $w$ is left maximum in $W / V$.

REMARK 4.3. Let $\mathscr{L}(W / V)$ be the set of reduced expressions for elements of $W / V$, viewed now as words in the alphabet $S$. If $x_{1} x_{2} \cdots x_{l}, y_{1} y_{2} \cdots y_{k} \in \mathscr{L}(W / V)$, 
and $l>k$, it follows by Theorem 4.2 that there exists a subword $z_{1} z_{2} \cdots z_{d}=$ $x_{i_{1}} x_{i_{2}} \cdots x_{i_{d}}, 1 \leq i_{1}<\cdots<i_{d} \leq l$, such that $z_{1} z_{2} \cdots z_{d} y_{1} y_{2} \cdots y_{k} \in \mathscr{L}(W / V)$ and $d \geq l-k$. This exchange property together with being suffix-hereditary makes $\mathscr{L}(W / V)$ into a special kind of formal language, called a strong greedoid. See [6] for a discussion of properties and other examples of such exchange languages.

The converse of Theorem 4.1 holds in finite $W$.

THEOREM 4.4. All lower left intervals in finite $W$ are generalized quotients of $W$. More precisely, if $W$ is finite and $u, v \in W$ where $u v=w_{0}$ then

$$
[e, u]_{L}=W /[e, v]_{R}=W /\{v\} .
$$

ProOF. It is easy to see that $W /[e, v]_{R}=W /\{v\}$.

We know that if $x y=w_{0}$ then $l(x)+l(y)=l\left(w_{0}\right)$, since $w_{0}$ is the left maximal element of $W$. Suppose now that $x y z=w_{0}$. It follows at once that

$$
l(x y)=l(x)+l(y) \Leftrightarrow l(y z)=l(y)+l(z) .
$$

This translates (for $u=x y$ and $v=z$ ) to the fact that if $u v=w_{0}$ then $[e, u]_{L}=$ $W /\{v\}$.

A subset $U \subseteq W$ will be called a prime quotient if $U=W /\{v\}$ for some $v \in W$. By Theorems 4.1 and 4.4 we have the following:

COROLlaRY 4.5. For finite $W$, the classes of generalized quotients, prime quotients, and lower left intervals coincide.

The following is a consequence of Theorem 4.4 and Proposition 2.3.

COROLLARY 4.6. In finite $W$, all left intervals are isomorphic to generalized quotients under both Bruhat order and left order.

We will now consider extensions of Corollary 4.5 to infinite $W$. We start with the relationship between generalized quotients and prime quotients. For any subset $V \subseteq W$ let $\bigvee V$ denote the right join of elements of $V$ when it exists. Note that since $W$ is a meet-semilattice, $\bigvee V$ exists if and only if $V$ has an upper bound in right order. This is always the case when $W$ is finite. We shall call $V$ right spherical if $V$ has an upper bound in right order.

THEOREM 4.7. If $V \subseteq W$ is right spherical then $W / V$ is a prime quotient which is equal to $W /\left\{v_{0}\right\}$ where $v_{0}=\bigvee V$.

Proof. It is easy to see that $W /\left\{v_{0}\right\} \subseteq W / V$. For the reverse inclusion we use induction on $l(w)$ to show that if $w \in W / V$ then $w \in W /\left\{v_{0}\right\}$. For $l(w)=0$ this holds trivially. Assume that $l(w)>0$. Then $w=s w^{\prime}$ where $l\left(w^{\prime}\right)=l(w)-1$ and $s \in S$. Since $w^{\prime}<_{L} w, w^{\prime} \in W / V$. Hence by induction $w^{\prime} \in W /\left\{v_{0}\right\}$. Now suppose that $w \notin W /\left\{v_{0}\right\}$. Then $\langle w\rangle\left\langle v_{0}\right\rangle$ is not reduced. This implies, by Lemma 2.4, that $s w^{\prime} v_{0}=w^{\prime} \widehat{v}_{0}$ where $\widehat{v}_{0}<_{B} v_{0}$ and $l\left(\widehat{v}_{0}\right)=l\left(v_{0}\right)-1$. Let $v \in V$. Since $v \leq_{R} v_{0}$, we have that $v_{0}=v y$ where $\langle v\rangle\langle y\rangle$ is reduced. It follows that $w^{\prime} \widehat{v}_{0}=s w^{\prime} v_{0}=s w^{\prime} v y=w^{\prime} v \widehat{y}$, where $\widehat{y}<_{B} y$, with the last equality following from Lemma 2.4 and the fact that $s\left\langle w^{\prime}\right\rangle\langle v\rangle$ is reduced. Hence $\widehat{v}_{0}=v \widehat{y}$ and $\langle v\rangle\langle\widehat{y}\rangle$ is reduced. Since $v$ was arbitrary, we have that $v \leq_{R} \widehat{v}_{0}$ for all $v \in V$. But this contradicts the fact that $v_{0}$ is the least upper bound of $V$ in right order. Hence $w \in W /\left\{v_{0}\right\}$, and we are done.

Theorem 4.7 gives a sufficient condition for a generalized quotient to be prime. We now show that for ordinary quotients this condition is also a necessary condition. 
THEOREM 4.8. Let $J \subseteq S$. Then $W^{J}$ is a prime quotient if and only if $W_{J}$ is finite.

PROOF. If $W_{J}$ is finite then $J$ is clearly right spherical and by Theorem $4.7, W^{J}$ is prime. Conversely, if $W^{J}$ is prime then $W^{J}=W /\{v\}$ for some $v \in W$. Since $s \notin W^{J}$ for all $s \in J, s v<_{L} v$. Recall that for all ordinary quotients, $W^{J}=W / W_{J}$ and $W=W^{J} \cdot W_{J}$. This is equivalent to the fact that $W^{J}$ is the set of minimal coset representatives modulo $W_{J}$. Left-right symmetry then implies that $v$ can be uniquely factored into $v=v_{1} v_{2}$ where

$$
v_{1} \in W_{J} \quad \text { and } \quad v_{2} \in J_{W}=\{w \in W \mid s w>w \text { for all } s \in J\}
$$

and $\left\langle v_{1}\right\rangle\left\langle v_{2}\right\rangle$ is reduced. Since $s v<_{L} v$ for all $s \in J$, it follows that $s v_{1}<_{L} v_{1}$ for all $s \in J$. Therefore by Proposition 2.1, $W_{J}$ is finite.

It turns out that when $W$ is infinite and $V \nsubseteq S, W / V$ can be prime for nonspherical $V$. This is verified by (ii) and (iii) of the following theorem.

THEOREM 4.9. Let $(W,\{a, b, c\})$ be the Coxeter group in which the orders of $a b, a c$, and $b c$ are $i, j$, and $k$, respectively, where $i, j, k \geq 3$. Then the following hold:

(i) The set $\{a b, c\}$ is not right spherical.

(ii) $W /\{a b c, b\}=W^{\{a, b\}}=W /\{a \vee b\}$.

(iii) If $i \geq 4$ then $\{a b c, b\}$ is not right spherical.

PrOOF. (i) Suppose that $\{a b, c\}$ is right spherical. We may assume without any loss of generality that $a b \vee c$ has minimum length among all $x y \vee z$ that exist, where $\{x, y, z\}=\{a, b, c\}$. Since $a, c \leq_{R} a b \vee c$ it follows that $a \vee c \leq_{R} a b \vee c$. Note that since $j \geq 3, a c a$ is reduced and $a c a \leq_{R} a \vee c$. Therefore, $a c a \leq_{R} a b \vee c$. This means that $a b \vee c=a c a w$ where $a c a\langle w\rangle$ is reduced, which implies that $a b<_{R} a c a w$. Consequently $b<_{R} c a w$. This implies that $c a \vee b \leq_{R} c a w$. But since $l(c a w)<$ $l(a b \vee c)$, the assumption that $a b \vee c$ has minimal length is contradicted. Hence $\{a b, c\}$ is not right spherical.

(ii) We clearly have $W /\{a b c, b\} \subseteq W^{\{a, b\}}$. Conversely, let $w \in W^{\{a, b\}}$. Then $\langle w\rangle a b$ is reduced. If $\langle w\rangle a b c$ is not reduced, then $w a b=w^{\prime} c$ where $\left\langle w^{\prime}\right\rangle c$ is reduced. This implies that $\{a b, c\}$ is left spherical, which contradicts (i). Hence $\langle w\rangle a b c$ is reduced. Since $\langle w\rangle b$ is also reduced, $w \in W /\{a b c, b\}$.

(iii) Suppose $\{a b c, b\}$ is right spherical. Then $b \leq_{R} a b c w$ for some $w \in W$ where $a b c\langle w\rangle$ is reduced. Since $a, b \leq_{R} a b c w$, it follows that $a \vee b \leq_{R} a b c w$. Since $i \geq 4$, $a b a b$ is reduced and $a b a b \leq_{R} a b c w$. Hence, $a b \leq_{R} c w$. This implies that $\{a b, c\}$ is right spherical, which contradicts (i).

Theorem 4.8 shows that the concept of generalized quotient is a more general concept than that of prime quotient. We now show that the concept of finite generalized quotient is less general than that of lower left interval. First we develop a simple test to determine whether or not a set is a generalized quotient.

For the discussion in this paragraph we shall let $L(V)$ and $R(V)$ denote the left and right generalized quotients determined by a subset $V \subseteq W$. By this we mean:

$$
L(V)=W / V
$$

and

$$
R(V)=V \backslash W=\{w \in W \mid l(v w)=l(v)+l(w) \text { for all } v \in V\}=\left(W / V^{-1}\right)^{-1}
$$


Directly from the definitions we have

$(\alpha)$ If $U \subseteq V$, then $L(U) \supseteq L(V)$ and $R(U) \supseteq R(V)$.

( $\beta) V \subseteq R(L(V))$ and $V \subseteq L(R(V))$, for all $V \subseteq W$.

This means that the pair of mappings $L, R: 2^{W} \rightarrow 2^{W}$ give a Galois connection on the Boolean lattice of all subsets of $W$. By well-known reasoning, see [3, p. 124], this implies that the mappings $V \rightarrow R L(V)$ and $V \rightarrow L R(V)$ are closure operations on $2^{W}$, i.e., in addition to property $(\beta)$, they are order preserving and idempotent. Furthermore, the mappings $L$ and $R$ are both order-reversing bijections between the $R L$-closed sets and the $L R$-closed sets. Now, clearly $U$ is $L R$-closed (i.e., $U=L R(U))$ if and only if $U=L(V)$ for some $V \subseteq W$, since $L(V)=L R L(V)$. Returning to the notation $W / V$ and $V \backslash W$ for left and right generalized quotients we have proven the following.

THEOREM 4.10. (i) A set $U$ is a left generalized quotient if and only if $U=$ $W /(U \backslash W)$.

(ii) There is a one-to-one correspondence between left generalized quotients $U$ and right generalized quotients $V$ given by $U=W / V$, or equivalently, $V=U \backslash W$.

Note that for finite Coxeter groups the correspondence between left and right generalized quotients (i.e., left and right lower intervals) is apparent already from Theorem 4.4.

We now apply the test, provided by Theorem 4.10(i), for determining whether or not a set is a generalized quotient, to the following example. Let $(W,\{a, b, c\})$ be the Coxeter group in which the orders of $a b, a c$, and $b c$ are $i, j$, and $k$, respectively, where $i, j, k \geq 3$. We shall show that $W_{\{a, b\}}$ is not a generalized quotient of $W$. We know that $W_{\{a, b\}} \backslash W=\{a, b\} W$. By Theorem 4.9(ii), cba $\in W /^{\{a, b\}} W$. Hence $W_{\{a, b\}} \neq W /\{a, b\} W=W /\left(W_{\{a, b\}} \backslash W\right)$. By Theorem 4.10(i), $W_{\{a, b\}}$ is then not a generalized quotient of $W$. However $W_{\{a, b\}}$ is a lower left interval of $W$. Hence not all lower left intervals are generalized quotients.

5. Alternative generalized quotients and convexity. Let $(W, S)$ be a Coxeter group and let $T$ be the set of conjugates of $S$. For any subset $A$ of $T$, define the set $W^{A}$ as in definition (1.3), i.e., $W^{A}=\left\{w \in W \mid w t>_{B} w\right.$ for all $t \in A\}$. We shall show that this alternative notion of generalized quotient is in fact more general than the generalized quotients that we have so far been considering in this paper. When $W$ is the symmetric group, the sets $W^{A}$ have an interesting interpretation as linear extensions of posets, see [10].

Recall that for all $w \in W, T_{w}=\left\{t \in T \mid t w<_{B} w\right\}$.

TheOREM 5.1. Let $V \subseteq W$. Then $W / V=W^{A}$ where $A=\bigcup_{v \in V} T_{v}$.

Proof. Let $w \in W / V$. If $w \notin W^{A}$ then there is some $v \in V$ and $t \in T_{v}$ such that $w t<_{B} w$. It follows that $l(w v)=l(w t t v) \leq l(w t)+l(t v)<l(w)+l(v)$. This contradicts the fact that $w \in W / V$. Hence, $w \in W^{A}$, and therefore, $W / V \subseteq W^{A}$.

Now let $w \in W^{A}$. Suppose that $w \notin W / V$. Then there is some $v \in V$ such that $\langle w\rangle\langle v\rangle$ is not reduced. Let $v=v_{1} s v_{2}$, where $\left\langle v_{1}\right\rangle s\left\langle v_{2}\right\rangle$ and $\langle w\rangle\left\langle v_{1}\right\rangle$ are reduced, but $\langle w\rangle\left\langle v_{1}\right\rangle s$ is not reduced. Then by Lemma $2.4, w v_{1} s=\widehat{w} v_{1}$, where $\widehat{w}<_{B} w$. We also have that $w v_{1} s=w t v_{1}$, where $t=v_{1} s v_{1}^{-1}$. Hence, $w t v_{1}=\widehat{w} v_{1}$, which means that $w t=\widehat{w}$. Since $w \in W^{A}$, it follows that $t \notin A$. This implies that $t \notin T_{v}$. By 
Proposition 2.5 we have $t \notin T_{v_{1} s}$, which is a contradiction since $t v_{1} s=v_{1}$. Hence, $w \in W^{A}$, and therefore, $W / V=W^{A}$.

Although there are lower left intervals of $W$ which are not of the form $W / V$ (cf. $\S 4)$, it happens that all lower left intervals are of the more general form $W^{A}$.

THEOREM 5.2. For any $w \in W,[e, w]_{L}=W^{A}$, where $A=T-T_{w^{-1}}$.

PROOF. This is a reformulation of Proposition 2.5.

We have chosen, in this paper, to define generalized quotients to be sets of the form $W / V$ rather than the more general form $W^{A}$, because we have been able to show that $W / V$ has a number of desirable properties, such as the chain property and having lexicographically shellable intervals under Bruhat order. It is an open question as to whether or not these properties also hold for $W^{A}$.

One general property that can be established for sets of the form $W^{A}$ is that of convexity under left order. A subset $U$ of a poset $P$ is said to be convex in $P$ if for all $u, w \in U$, every minimum length path from $u$ to $w$ in the Hasse diagram of $P$ is in $U$. For left order this is equivalent to Tits' [22] notion of convexity in Coxeter complexes.

It is a consequence of Theorem 2.19 in [22] that the sets $W^{A}$ are precisely the convex order ideals in $W$ (under left order), and their translates $W^{A} \cdot w, w \in W$, are precisely the convex subsets in $W$. Tits' theorem can be formulated as follows.

For any subsets $A$ and $D$ of $T$, define $W_{D}^{A}$ to be the set

$$
W_{D}^{A}=\left\{w \in W \mid t \in D \Rightarrow w t<_{B} w \text { and } t \in A \Rightarrow w t>_{B} w\right\} .
$$

THEOREM 5.3 (TITS). A subset $C$ of $W$ is convex (for left order) if and only if $C=W_{D}^{A}$ for some subsets $A, D \subseteq T$. In particular, $C$ is a convex order ideal if and only if $C=W^{A}$ for some $A \subseteq T$.

In an appendix (\$10) we will discuss convexity from an order-theoretic point of view. A direct proof (not via Coxeter complexes) of Theorem 5.3 is given there. Of interest now is the following consequence.

COROLLARY 5.4. Every generalized quotient $W / V$ is a convex order ideal under left order.

6. Descent classes. For $K \subseteq S$ the descent class $D_{K}$ is defined to be the set $\left\{w \in W \mid w s<_{R} w\right.$ if and only if $\left.s \in K\right\}$. More generally, for $I \subseteq J \subseteq S$ define the descent class $D_{I}^{J}$ to be $\bigcup_{I \subseteq K \subseteq J} D_{K}$. Note that $W^{J}=D_{\varnothing}^{\bar{J}}$, where $\bar{J} \operatorname{denotes} S-J$. Hence the ordinary quotients form a subset of the descent classes. In the symmetric group $\mathscr{S}_{n}$, the descent class $D_{K}$ is the set of permutations whose descents (in the usual sense) occur in the positions which correspond to elements of $K$.

THEOREM 6.1. For any $I \subseteq J \subseteq S$ such that $W_{I}$ is finite, the descent class $D_{I}^{J}$ is isomorphic to a generalized quotient under Bruhat order and left order.

Proof. Let $V=\left\{w_{0}(I)\right\} \cdot \bar{J}$. We claim that $W / V$ is isomorphic to $D_{I}^{J}$ under both Bruhat order and left order. The isomorphism $\varphi: W / V \rightarrow D_{I}^{J}$ is given by $\varphi(w)=w w_{0}(I)$.

First we show that $\varphi(w) \in D_{I}^{J}$. Since $\langle w\rangle\langle v\rangle$ is reduced for all $v \in V$ and $w_{0}(I) \leq_{R} v,\langle w\rangle\left\langle w_{0}(I)\right\rangle$ is reduced. Hence for all $s \in I, w w_{0}(I) s<_{R} w w_{0}(I)$. We 
now have

$$
\varphi(w) s<_{R} \varphi(w), \quad \text { for all } s \in I .
$$

For all $s \in \bar{J},\langle w\rangle\left\langle w_{0}(I)\right\rangle s$ is reduced, since $\left\langle w_{0}(I)\right\rangle_{s}$ is reduced and $w_{0}(I) s \in V$. Hence

$$
\varphi(w) s>_{R} \varphi(w), \quad \text { for all } s \in \bar{J}
$$

It follows from (6.1) and (6.2) that $\varphi(w) \in D_{I}^{J}$.

The map $\varphi$ is clearly injective. We now show that $\varphi$ is also surjective. Let $w \in D_{I}^{J}$. We can decompose $w$ into $w=u v$ where $u \in W^{I}$ and $v \in W_{I}$. To show that $\varphi$ is surjective it suffices to show that $v=w_{0}(I)$. Since $w s<_{R} w$ for all $s \in I$, $\langle u\rangle\langle v\rangle s$ is not reduced. It follows that $v s<_{R} v$ for all $s \in I$. By Proposition 2.1, $W_{I}$ is finite and $v=w_{0}(I)$.

The fact that $\varphi$ and its inverse are order preserving under Bruhat order follows from Lemma 2.2. Under left order it is trivial that $\varphi$ and its inverse are order preserving.

THEOREM 6.2. Let $I \subseteq J \subseteq S$.

(1) If $D_{I}^{J}$ is nonempty then $W_{I}$ is finite and

$$
D_{I}^{J}=\left\{w \in W^{\bar{J}} \mid w \geq_{L} w_{0}(I)\right\} .
$$

(2) If $D_{I}^{J}$ is finite and nonempty then $W_{I}$ and $W^{\bar{J}}$ are finite and

$$
D_{I}^{J}=\left[w_{0}(I), w_{0}^{\bar{J}}\right]_{L}
$$

where $w_{0}^{\bar{J}}$ is the maximum element of $W^{\bar{J}}$.

PROOF. (1) It follows from the part of the proof of Theorem 6.1 in which it is shown that $\varphi$ is surjective that if $D_{I}^{J}$ is nonempty then $W_{I}$ is finite and all $w \in D_{I}^{J}$ satisfy $w \geq_{L} w_{0}(I)$. Clearly we also have that $D_{I}^{J} \subseteq W^{\bar{J}}$. Hence,

$$
D_{I}^{J} \subseteq\left\{w \in W^{\bar{J}} \mid w \geq_{L} w_{0}(I)\right\} .
$$

Conversely, let $w \in W^{\bar{J}}$ and $w \geq_{L} w_{0}(I)$. Then $w=u w_{0}(I)$, where $\langle u\rangle\left\langle w_{0}(I)\right\rangle$ is reduced. If $s \in I$ then $w s=u w_{0}(I) s<_{R} u w_{0}(I)=w$. If $s \in \bar{J}$ then $w s>_{R} w$, since $w \in W^{\bar{J}}$. Hence, $w \in D_{I}^{J}$.

(2) It follows from (1) that if $D_{I}^{J}$ is finite then $D_{I}^{J}$ has an element which is a left maximal element of $W^{\bar{J}}$. By Theorem $4.1, W^{\bar{J}}$ is finite and $W^{\bar{J}}=\left[e, w_{0}^{\bar{J}}\right]_{L}$. The result now follows from (1).

Descent classes can be decomposed into smaller subsets which are also isomorphic to generalized quotients. For $I \subseteq J \subseteq S$ and $b \in D_{I} \cap W_{J}$ let

$$
E_{I J}(b)=\left\{w \in D_{I} \mid w=a b \text { for some } a \in W^{J}\right\} .
$$

THEOREM 6.3. For $I \subseteq J \subseteq S, D_{I}=\sum_{b \in D_{I} \cap W_{J}} E_{I J}(b)$. (The symbol $\sum$ denotes disjoint union.)

Proof. Clearly we have $D_{I} \supseteq \bigcup_{b \in D_{I} \cap W_{J}} E_{I J}(b)$. Conversely, let $w \in D_{I}$. We can decompose $w$ into $w=a b$ where $a \in W^{J}$ and $b \in W_{J}$. If $b s>_{R} b$ for some $s \in I$ then $a b s>_{R} a b$ since $a \in W^{J}$ and $b s \in W_{J}$. This contradicts $a b=w \in D_{I}$. 
Hence $b s<_{R} b$ for all $s \in I$. Another consequence of $a b \in D_{I}$ is that $a b s>_{R} a b$ for all $s \in \bar{I}$. This implies that $b s>_{R} b$ for all $s \in \bar{I}$. Hence $b \in D_{I}$. It follows that $w \in E_{I J}(b)$. Hence, $D_{I}=\bigcup_{b \in D_{I} \cap W_{J}} E_{I J}(b)$.

To show that $E_{I J}\left(b_{1}\right) \cap E_{I J}\left(b_{2}\right) \neq \varnothing$ implies $b_{1}=b_{2}$, let $w \in E_{I J}\left(b_{1}\right) \cap E_{I J}\left(b_{2}\right)$. Then $w=a_{1} b_{1}=a_{2} b_{2}$, where $a_{1}, a_{2} \in W^{J}$. Since every element of $W$ decomposes uniquely as a product of elements from $W^{J}$ and $W_{J}, a_{1}=a_{2}$ and $b_{1}=b_{2}$.

THEOREM 6.4. For $I \subseteq J \subseteq S$ and $b \in D_{I} \cap W_{J}, E_{I J}(b)$ is isomorphic to a generalized quotient under Bruhat order and left order.

ProOF. Let $V=J \cap\{b\} \cdot \bar{I}$. We claim that the map $\varphi: W / V \rightarrow E_{I J}(b)$ defined by $\varphi(a)=a b$ is an isomorphism under Bruhat order and left order. The verification parallels the proof of Theorem 6.1 and is left to the reader.

We can now conclude that each set $J$ containing $I$ induces a partition of $D_{I}$ into isomorphs of generalized quotients. This partition will be called a J-partition of $D_{I}$. The $J$-partitions of $D_{I}$ have the following relationship.

THEOREM 6.5. If $I \subseteq J_{1} \subseteq J_{2} \subseteq S$ then the $J_{1}$-partition of $D_{I}$ is coarser than the $J_{2}$-partition of $D_{I}$.

PROOF. Let $E_{I J_{2}}\left(b_{2}\right)$ be a block of the $J_{2}$-partition of $D_{I}$. Since $b_{2} \in D_{I}$, $b_{2}$ belongs to a block, say $E_{I J_{1}}\left(b_{1}\right)$, of the $J_{1}$-partition of $D_{I}$. We claim that $E_{I J_{2}}\left(b_{2}\right) \subseteq E_{I J_{1}}\left(b_{1}\right)$, which proves the theorem. The routine verification is left to the reader.

The extremal $J$-partitions of $D_{I}$ are obtained when $J=I$ and $J=S$. Since $D_{I} \cap W_{I}=\left\{w_{0}(I)\right\}$, the $I$-partition of $D_{I}$ consists of a single block $E_{I I}\left(w_{0}\right)=D_{I}$. Since $E_{I S}(b)=\{b\}$ for all $b \in D_{I} \cap W_{S}=D_{I}$, the $S$-partition of $D_{I}$ is the partition $\sum_{b \in D_{I}}\{b\}$.

We now present a relationship between the blocks of a $J$-partition of $D_{I}$. A Bruhat order preserving function $\varphi: A \rightarrow B$, where $A, B \subseteq W$ is said to be increasing if $a \leq_{B} \varphi(a)$ for all $a \in A$. Let $P$ be a ranked poset with a minimum element $\hat{0}$. A subposet $Q$ of $P$ shall be called a semi-ideal of $P$ if $Q$ contains $\hat{0}$ and is ranked with rank function the restriction of the rank function of $P$. Note that any order ideal of $P$ is a semi-ideal of $P$.

THEOREM 6.6. Let $E_{I J}\left(b_{1}\right)$ and $E_{I J}\left(b_{2}\right)$ be blocks of a J-partition of $D_{I}$. Then there is an increasing isomorphism under Bruhat order between $E_{I J}\left(b_{1}\right)$ and a Bruhat semi-ideal of $E_{I J}\left(b_{2}\right)$ if and only if $b_{1} \leq_{B} b_{2}$.

PROOF. $(\Rightarrow)$ Since $b_{1}$ and $b_{2}$ are the minimum elements of $E_{I J}\left(b_{1}\right)$ and $E_{I J}\left(b_{2}\right)$ respectively, and the isomorphism is increasing, it is immediate that $b_{1} \leq_{B} b_{2}$.

$(\Leftarrow)$ This is a special case of the following lemma.

LEMMA 6.7. Let $I_{1}, I_{2} \subseteq J, b_{1} \in D_{I_{1}} \cap W_{J}$, and $b_{2} \in D_{I_{2}} \cap W_{J}$. If $b_{1} \leq_{B} b_{2}$ then the function $\varphi: E_{I_{1} J}\left(b_{1}\right) \rightarrow E_{I_{2} J}\left(b_{2}\right)$ defined by $\varphi\left(a b_{1}\right)=a b_{2}$ is an increasing isomorphism under Bruhat order between $E_{I_{1} J}\left(b_{1}\right)$ and a Bruhat semi-ideal of $E_{I_{2} J}\left(b_{2}\right)$.

PROOF. We may assume that $b_{1}<_{B} b_{2}$. To show that $a b_{2} \in E_{I_{2} J}\left(b_{2}\right)$, it suffices to show that $a b_{2} \in D_{I_{2}}$. If $s \in I_{2}$ then $a b_{2} s=a \widehat{b}_{2}$ where $\widehat{b}_{2}<_{B} b_{2}$. Since $a \in W^{J}$ and $b_{2} \in W_{J}$, it follows that $a b_{2} s<_{R} a b_{2}$. 
If $s \in J-I_{2}$ then $b_{2} s>_{R} b_{2}$. Again, since $a \in W^{J}$ and $b_{2} s \in W_{J}$, we have $a b_{2} s>_{R} a b_{2}$.

Now suppose that $s \in \bar{J}$. Assume that $a b_{2} s<_{R} a b_{2}$. Since $a b_{1} \in D_{I_{1}}, a b_{1} s>_{R}$ $a b_{1}$. We also have by Lemma 2.2 that $a b_{1}<_{B} a b_{2}$. It now follows from the lifting property that $a b_{1} \leq_{B} a b_{2} s$. Since $b_{2} \in D_{I_{2}}$, Lemma 2.4 gives that $a b_{2} s=\widehat{a} b_{2}$ where $\widehat{a}<_{B} a$. Hence $a b_{1} \leq_{B} \widehat{a} b_{2}$. It follows from the subword property that $a b_{1}=\tilde{a} \widehat{b}_{2}$ where $\tilde{a}<_{B} a$ and $\widehat{b}_{2} \leq b_{2}$. Let $\tilde{a}=a_{1} a_{2}$ where $a_{1} \in W^{J}$ and $a_{2} \in W_{J}$. Then $a_{1} a_{2} \widehat{b}_{2}=a b_{1}$. By uniqueness of the decomposition, $a=a_{1}$, which is a contradiction since $a_{1} \leq_{R} \tilde{a}<_{B} a$. Consequently, $a b_{2} s>_{R} a b_{2}$. We may now conclude that $a b_{2} \in D_{I_{2}}$.

Clearly, $\varphi$ is injective, increasing, and rank preserving. It follows from Lemma 2.2 that $\varphi$ and its inverse are Bruhat order preserving. This and the fact that $\varphi$ is rank preserving implies that $\varphi\left(E_{I_{1} J}\left(b_{1}\right)\right)$ is a Bruhat semi-ideal of $E_{I_{2} J}\left(b_{2}\right)$.

Another consequence of Lemma 6.7 is Theorem 6.9 below. First we need another lemma, due to Deodhar [13].

LEMMA 6.8. The map $\pi: W \rightarrow W^{J}$ defined by $\pi(w)=u$, where $w$ decomposes into $w=u v$ with $u \in W^{J}$ and $v \in W_{J}$, is a Bruhat order preserving map.

ProOF. Let $w_{1}, w_{2} \in W$ and $w_{1}<_{B} w_{2}$. Suppose that $w_{1}$ decomposes into $u_{1} v_{1}$ and $w_{2}$ decomposes into $u_{2} v_{2}$ where $u_{1}, u_{2} \in W^{J}$ and $v_{1}, v_{2} \in W_{J}$. Then $\pi\left(w_{1}\right)=u_{1}$ and $\pi\left(w_{2}\right)=u_{2}$. If $w_{1} \leq w_{2}$ then by the subword property $u_{1} v_{1}=\widehat{u}_{2} \widehat{v}_{2}$, where $\widehat{u}_{2} \leq_{B} u_{2}$ and $\widehat{v}_{2} \leq_{B} v_{2}$. Let $\widehat{u}_{2}=a b$ where $a \in W^{J}$ and $b \in W_{J}$. Then $u_{1} v_{1}=a b \widehat{v}_{2}$. By the uniqueness of the decomposition $u_{1}=a$. Hence, $u_{1} \leq_{B} u_{2}$. It follows that $\pi$ is Bruhat order preserving.

THEOREM 6.9. Let $I \subset J \subseteq S$, and suppose that $D_{J}$ is nonempty. Then the map $\psi: D_{I} \rightarrow D_{J}$ defined by $\psi(w)=a w_{0}(J)$, where $w$ decomposes into $w=$ ab with $a \in W^{J}$ and $b \in W_{J}$, is an increasing Bruhat order preserving map from $D_{I}$ onto a Bruhat semi-ideal of $D_{J}$.

PROOF. Since $D_{J}$ is nonempty, by Theorem $6.2 W_{J}$ is finite and therefore $W_{J} \cap D_{I}$ is finite. Since $W_{J} \cap D_{I}$ is a descent class of $W_{J}$, by Theorem $6.2 W_{J} \cap D_{I}$ has a maximum element $b_{0}$. By Lemma 6.7 , for each $b \in W_{J} \cap D_{I}$, the map $\varphi_{b}: E_{I J}(b) \rightarrow$ $E_{I J}\left(b_{0}\right)$ defined by $\varphi_{b}(a b)=a b_{0}$ is an increasing map. Define $\varphi: D_{I} \rightarrow E_{I J}\left(b_{0}\right)$ to be the join of the maps $\left\{\varphi_{b} \mid b \in W_{J} \cap D_{I}\right\}$, i.e., $\varphi(w)=\varphi_{b}(w)$ for $w \in E_{I J}(b)$. Clearly, $\varphi$ is increasing since each $\varphi_{b}$ is increasing. Note also that $\varphi$ is surjective. It follows from Lemmas 2.2 and 6.8 that $\varphi$ is a Bruhat order preserving map.

Now by Lemma 6.7 there is an increasing Bruhat order preserving map $\gamma$ from $E_{I J}\left(b_{0}\right)$ onto a Bruhat semi-ideal of $E_{J J}\left(w_{0}(J)\right)=D_{J}$. The composition $\gamma \circ \varphi$ is an increasing Bruhat order preserving map from $D_{I}$ onto a Bruhat semi-ideal of $D_{J}$. Note that the composition $\gamma \circ \varphi$ is precisely $\psi$ defined above.

A subposet $Q$ of a poset $P$ is said to be a retract of $P$ if there is an order preserving map from $P$ to $Q$ whose restriction to $Q$ is the identity map. Note that according to Lemma $6.8, W^{J}$ is a retract of $W$ under Bruhat order.

THEOREM 6.10. For any $J \subseteq S$ such that $D_{J}$ is nonempty, $D_{J}$ is a retract of $W$ under Bruhat order.

PROOF. Let $\pi: W \rightarrow W^{\bar{J}}$ be the retraction map as in Lemma 6.8. Since $W^{\bar{J}}=$ $D_{\varnothing}^{J}=\sum_{I \subseteq J} D_{I}$, let $\varphi: W^{\bar{J}} \rightarrow D_{J}$ be the join of the maps given in Theorem 6.9 . 
Then $\varphi(a b)=a w_{0}(J)$ where $a \in W^{J}$ and $b \in W_{J}$. Clearly, $\varphi$ is the identity on $D_{J}$, and by Lemma 6.8, $\varphi$ is Bruhat order preserving. Hence, the composition $\varphi \circ \pi$ is a Bruhat order preserving map of $W$ onto $D_{J}$, whose restriction to $D_{J}$ is the identity map.

7. Tableau quotients in the symmetric group. Recall that for the symmetric group $\mathscr{S}_{n}$ of permutations of the set $\{1,2, \ldots, n\}$, the standard choice for a set $S$ of Coxeter generators is the set of adjacent transpositions $(i, i+1)$, and the set $T$ of conjugates of $S$ is the set of all transpositions $(i, j), 1 \leq i<j \leq n$. We shall think of permutations in $\mathscr{S}_{n}$ as words with $n$ distinct letters $1,2, \ldots, n$. If $w \in \mathscr{S}_{n}$ and $t$ is the transposition $(i, j)$ then the permutation $t w$, obtained from $w$ by transposing letter $i$ and letter $j$ in $w$, is greater than $w$ in Bruhat order if and only if $i<j$ and $i$ appears to the left of $j$ in $w$. Going "up" in left order requires transposing consecutive letters and going "up" in right order requires transposing letters in adjacent positions, where the smaller letter is on the left in both cases.

We now consider two natural partial orderings on the set of standard tableaux of a fixed shape, one of which is isomorphic to Bruhat order and the other to left order on a generalized quotient in the symmetric group. Let $\mu=\left(\mu_{1} \geq \mu_{2} \geq \cdots \geq \mu_{k}\right)$ and $\lambda=\left(\lambda_{1} \geq \lambda_{2} \geq \cdots \geq \lambda_{k}\right)$ be partitions of $m$ and $m+n$, respectively, where $0 \leq \mu_{i} \leq \lambda_{i}$ for $i=1,2, \ldots, k$. A tableau of skew shape $\lambda / \mu$ is an array of integers, each integer $1,2, \ldots, n$ appearing exactly once, such that for each $i=1,2, \ldots, k$, row $i$ consists of $\mu_{i}$ blanks followed by $\lambda_{i}-\mu_{i}$ integers. A standard tableau is a tableau in which every row (from left to right) and every column (from top to bottom, where row 1 is the top row) is increasing (cf. [17, §5.1.4]). Let $\mathscr{T}_{\lambda / \mu}$ be the set of standard tableaux of shape $\lambda / \mu$. We define Bruhat order on $\mathscr{T}_{\lambda / \mu}$ to be the order relation generated by $T<T^{\prime}$ if $T^{\prime}=(i, j) T$, where $1 \leq i<j \leq n$, entry $i$ appears above entry $j$ in $T$, and the transposition $(i, j)$ acts on $T$ by transposing entries $i$ and $j$. Left order is defined similarly except that $j=i+1$. The Hasse diagram for Bruhat and left order on $\mathscr{T}_{\lambda / \mu}$, where $\lambda=(3,2,1)$ and $\mu=(1,0,0)$, is given in Figure 7.1 (the solid lines represent left order and the dotted lines represent the additional relations of Bruhat order).

The standard tableau of shape $\lambda / \mu$ whose first row contains $1,2, \ldots, \lambda_{1}-\mu_{1}$, whose second row contains $\lambda_{1}-\mu_{1}+1, \lambda_{1}-\mu_{1}+2, \ldots, \lambda_{1}-\mu_{1}+\lambda_{2}-\mu_{2}$, etc., shall be referred to as the row tableau of shape $\lambda / \mu$. The column tableau of shape $\lambda / \mu$ is defined similarly.

THEOREM 7.1. Let $\lambda / \mu$ be any skew shape.

(1) Both Bruhat order and left order on $\mathscr{T}_{\lambda / \mu}$ are graded posets, whose minimum element is the row tableau of shape $\lambda / \mu$, and whose maximum element is the column tableau of shape $\lambda / \mu$.

(2) Left order on $\mathscr{T}_{\lambda / \mu}$ is a lattice.

(3) Bruhat order on $\mathscr{T}_{\lambda / \mu}$ is CL-shellable.

ProOF. We will show in Theorem 7.5 that $\mathscr{T}_{\lambda / \mu}$ is isomorphic, under both Bruhat order and left order, to a generalized quotient in the symmetric group $\mathscr{S}_{n}$. The result will then follow from Corollary 3.9 and Theorem 4.1.

Theorem 7.1 actually holds for shapes more general than skew shapes. See Remark 7.3 about this. 


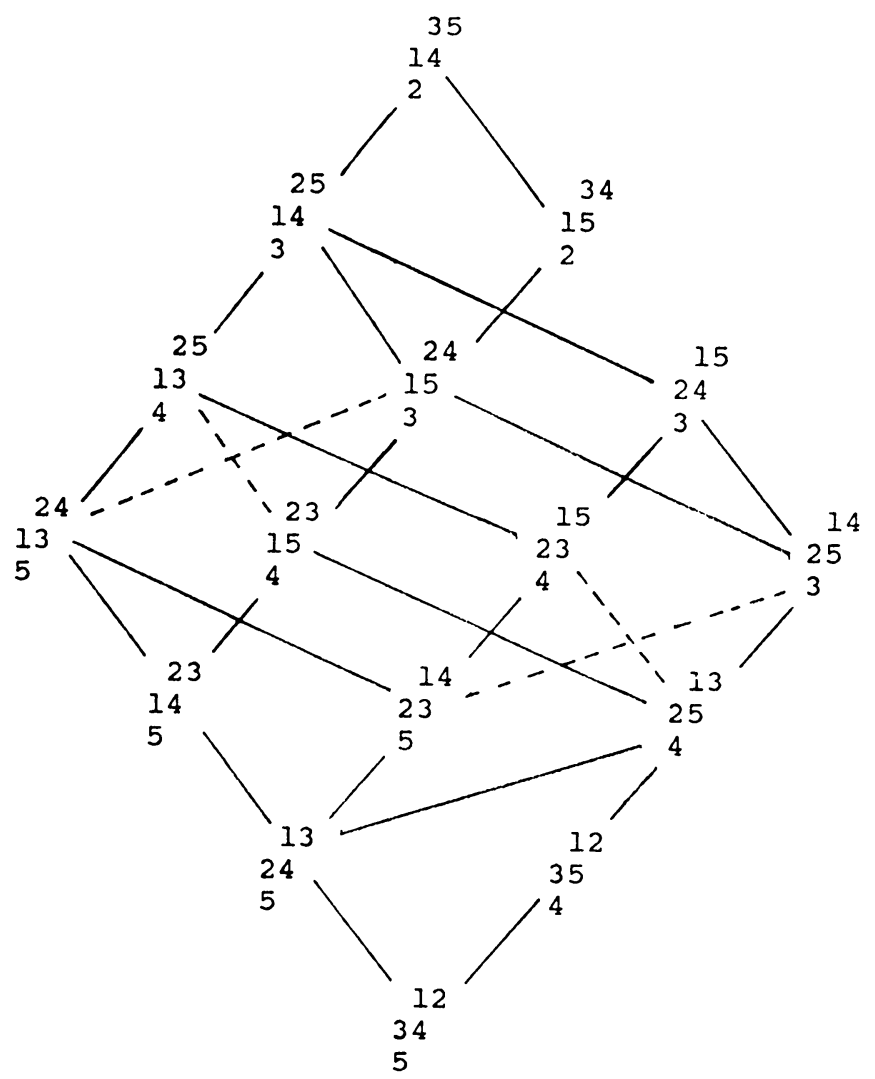

FIGURE 7.1

We shall now describe four maps from $\mathscr{T}_{\lambda / \mu}$ onto left intervals of $\mathscr{S}_{n}$ which are either isomorphisms or anti-isomorphisms under Bruhat order and left order. The word of a tableau $T$, denoted by $w(T)$, is the permutation in $\mathscr{S}_{n}$ obtained by reading the entries of $T$ in some specified order which we call a reading order. We shall consider four different reading orders. Let $T$ be the tableau

$$
\begin{aligned}
& 134 \\
& 26 \\
& 5
\end{aligned}
$$

(1) In row order the tableau entries are read row by row from left to right and from top to bottom; and $w(T)$ is 134265 .

(2) In column order the tableau entries are read column by column from top to bottom and from left to right; and $w(T)$ is 125364 .

(3) In French order the tableau entries are read row by row from left to right and from bottom to top; and $w(T)$ is 526134 .

(4) In Hebrew order the tableau entries are read row by row from right to left and from top to bottom; and $w(T)$ is 431625 . 
THEOREM 7.2. Let $w\left(\mathscr{T}_{\lambda / \mu}\right)$ be the set $\left\{w(T) \mid T \in \mathscr{T}_{\lambda / \mu}\right\}$ where $w$ is some fixed reading order. If the reading order is row order or column order then $w\left(\mathscr{T}_{\lambda / \mu}\right)$ is a generalized quotient and hence, a lower left interval of $\mathscr{S}_{n}$.

PROOF. Since the result for row order is equivalent to the result for column order (by considering the conjugate shape), we need only prove it for row order. We label the "squares" of the shape $\lambda / \mu$ in row order. For $i=1,2, \ldots, n$, let $r_{i}$ be the label of the right-most square in the row of the square labeled $i$ and let $b_{i}$ be the label of the square directly below square $i$, unless square $i$ has nothing below it, in which case $b_{i}=0$. For each $i$ such that $b_{i} \neq 0$, let $v_{i}$ be the permutation

$$
\begin{aligned}
(i, i+1) & (i+1, i+2) \cdots\left(r_{i}-1, r_{i}\right) \\
& \cdot\left(b_{i}-1, b_{i}\right)\left(b_{i}-2, b_{i}-1\right) \cdots\left(r_{i}+1, r_{i}+2\right)\left(r_{i}, r_{i}+1\right) .
\end{aligned}
$$

Let $T$ be a tableau of shape $\lambda / \mu$. Note that the permutation $w(T) v_{i}$ is obtained from the permutation $w(T)$ by first moving the entry of square $i$ to the right until it reaches the end of its row; and then moving the entry of square $b_{i}$ to the left until it reaches the beginning of its row; and finally interchanging these two entries, which are now in positions $r_{i}$ and $r_{i}+1$. It follows that $\langle w(T)\rangle\left\langle v_{i}\right\rangle$ is reduced if and only if all the entries to the right of square $i$ are greater than that of square $i$, all the entries to the left of square $b_{i}$ are less than that of square $b_{i}$, and the entry of square $i$ is less than that of square $b_{i}$.

Let $J$ be the set $\left\{(i, i+1) \mid i=1,2, \ldots, n-1, i \neq r_{i}\right\}$. Note that $J$ is the set of all transpositions that corrrespond to pairs of horizontally adjacent squares in a tableau of shape $\lambda / \mu$. Hence, if $T$ is a tableau of shape $\lambda / \mu$, then $\langle w(T)\rangle s$ is reduced for all $s \in J$ if and only if the rows of $T$ are increasing from left to right.

Now let $V=J \cup\left\{v_{i} \mid i=1,2, \ldots, n, b_{i} \neq 0\right\}$. It follows from the above analysis that $w\left(\mathscr{T}_{\lambda / \mu}\right)=W / V$. By Theorem $4.1, w\left(\mathscr{T}_{\lambda / \mu}\right)$ is a lower left interval.

REMARK 7.3 The preceding result is true in greater generality. Let $\Gamma$ be any set of $n$ squares from a sufficiently large chessboard. Say that a filling of the squares of $\Gamma$ with the integers $1,2, \ldots, n$, each occurring once, is a standard tableau of shape $\Gamma$, if each row is increasing (also across gaps) and each consecutive segment of each column is increasing. For instance, the following is a standard tableau:

15

26

347

Let $\mathscr{T}_{\Gamma}$ be the set of standard tableaux of shape $\Gamma$ and let $w(\mathscr{T})$ be the set of tableau words read in row order. The proof of Theorem 7.2 shows that $w\left(\mathscr{T}_{\Gamma}\right)$ is a generalized quotient, and hence a lower left interval of $\mathscr{S}_{n}$. In Theorem 7.6 a much more general situation will be described. For simplicity we will, in the sequel, discuss only the skew shapes $\lambda / \mu$ previously defined, although the results hold for general shapes. Recently, R. Dipper and G. James [14, Lemma 1.5] have also shown that the set of standard tableau words read in row order gives a lower left interval.

REMARK 7.4. The fact that $w\left(\mathscr{T}_{\lambda / \mu}\right)$ is a lower left interval has an algorithmic aspect. Say we want to generate all standard tableaux of shape $\lambda / \mu$ listed in order of the number of inversions of their tableau words read in row order. An algorithm for doing this can be described as follows: Start with the column tableau $T_{1}$, which is the maximum element of $\mathscr{T}_{\lambda / \mu}$. Suppose all the standard tableaux whose row 
words have $p$ inversions have already been generated, where $0<p \leq l\left(w\left(T_{1}\right)\right)$. Then for each such tableau $T$ and each entry $i$ for which $i+1$ is in a higher row than $i$ in $T$, transpose $i$ and $i+1$ to obtain a tableau whose row word has $p-1$ inversions. After deleting duplications, this will have generated a complete list of all $T \in \mathscr{T}_{\lambda / \mu}$ such that $w(T)$ has $p-1$ inversions. The sequence of standard tableaux produced by this algorithm is clearly a linear extension of the dual of Bruhat order on $\mathscr{T}_{\lambda / \mu}$. An algorithm of Nijenhuis and Wilf [18] also generates a sequence of standard tableaux which happens to be a linear extension of the dual of Bruhat order on $\mathscr{T}_{\lambda / \mu}$.

The following theorem includes Theorem 7.2 and provides an explicit isomorphism or anti-isomorphism between the posets $\mathscr{T}_{\lambda / \mu}$ and $w\left(\mathscr{T}_{\lambda / \mu}\right)$ for all the abovementioned reading orders.

THEOREM 7.5. Let $w: \mathscr{T}_{\lambda / \mu} \rightarrow \mathscr{S}_{n}$ be the map in which $w(T)$ is the word of $T$ obtained from some fixed reading order. If the reading order is row order or Hebrew order, then $w$ is an isomorphism, under Bruhat order and left order, between $\mathscr{T}_{\lambda / \mu}$ and a left interval of $\mathscr{S}_{n}$. If the reading order is column order or French order, then $w$ is an anti-isomorphism, under Bruhat order and left order, between $\mathscr{T}_{\lambda / \mu}$ and a left interval of $\mathscr{S}_{n}$. Moreover, the left interval is a lower left interval when the reading order is row order or column order.

In order to show that the image of $\mathscr{T}_{\lambda / \mu}$ under each of the maps is a left interval we shall rely on a general result presented below. We define a tableau quotient to be the image of $\mathscr{T}_{\lambda / \mu}$ under any of the maps in Theorem 7.5. The ordinary quotients $W^{J}$ in $W=\mathscr{S}_{n}$ form a subclass of the tableau quotients. Indeed, the ordinary quotients are of the form $w\left(\mathscr{T}_{\lambda / \mu}\right)$ where $\lambda / \mu$ consists of nonoverlapping rows and the reading order is row order, column order, or French order. The descent classes $D_{I}$ in $\mathscr{S}_{n}$ are also tableau quotients. Here $\lambda / \mu$ has zigzag shape and the reading order is French order or Hebrew order. Note that the poset in Figure 7.1 corresponds to a descent class. More generally, descent classes $D_{I}^{J}$ in $\mathscr{S}_{n}$ are tableau quotients, corresponding to shapes where the rows overlap in at most one square, and the reading order is French or Hebrew order. Garsia and Remmel [16] show that tableau quotients, for French order and Hebrew order, are unions of dual Knuth equivalence classes.

Tableau quotients form a special case of a more general structure. Let $P$ be a poset with $n$ elements. A standard labeling $L$ of $P$ is a labeling of $P$ with integers, each integer $1,2, \ldots, n$ appearing exactly once, such that the labeling is compatible with the partial ordering, i.e., if $x<_{P} y$ then $L(x)<L(y)$. If we specify an ordering of the elements of $P, x_{1}, x_{2}, \ldots, x_{n}$, and then read the standard labeling $L$ in this specified order, we obtain a permutation $L\left(x_{1}\right), L\left(x_{2}\right), \ldots, L\left(x_{n}\right)$ in $\mathscr{S}_{n}$. We shall call the specified linear order, a reading order. If $\pi$ is a reading order of $P$ then we let $\mathscr{L}(P, \pi)$ denote the set of permutations obtained by reading standard labelings of $P$ in the order given by $\pi$.

The set of standard tableaux of a fixed shape clearly is a set of standard labelings of a poset. Each of the four orders, row order, column order, French order, and Hebrew order, are examples of reading orders of the poset. The sets $w\left(\mathscr{T}_{\lambda / \mu}\right)$, in Theorem 7.5, are hence of the form $\mathscr{L}(P, \pi)$. We now present a simple criterion on $\pi$ for determining whether or not $\mathscr{L}(P, \pi)$ is a left interval of $\mathscr{S}_{n}$. 
THEOREM 7.6. Let $P$ be a poset with $n$ elements and let $\pi$ be a reading order of $P$. Then $\mathscr{L}(P, \pi)$ is a left interval of $\mathscr{S}_{n}$ if and only if $\pi$ satisfies the following condition.

(*) For all $x<_{P} z$ and $y \in P$, if $x$ precedes $y$ which precedes $z$ in $\pi$, or if $z$ precedes $y$ which precedes $x$ in $\pi$, then $x<_{P} y$ or $y<_{P} z$.

The proof of Theorem 7.6 will appear in [10]. It is also observed in [10] that the class of posets which admit reading orders satisfying condition $(*)$ is precisely the class of two-dimensional posets. In fact, it is shown that every left interval of $\mathscr{S}_{n}$ is of the form $\mathscr{L}(P, \pi)$ where $P$ is two-dimensional and $\pi$ satisfies condition (*).

PROOF OF THEOREM 7.5. It is easy to check that row order, column order, French order, and Hebrew order are reading orders which satisfy $(*)$ of Theorem 7.6. Hence, $w\left(\mathscr{T}_{\lambda / \mu}\right)$ is a left interval for these orders. Clearly, $w\left(\mathscr{T}_{\lambda / \mu}\right)$ contains the identity when the reading order is row or column order. Hence, the left intervals are lower left intervals in these cases.

It is easy to see that the map $w$ is Bruhat and left order preserving when the reading order is row order or Hebrew order, and $w$ is Bruhat and left order reversing when the reading order is column order or French order. It is also immediate that the inverse of $w$ on $w\left(\mathscr{T}_{\lambda / \mu}\right)$ is left order preserving or reversing. For Bruhat order this is not immediate. Let us restrict ourselves to the case that the reading order is row order or Hebrew order. The case of column order and French order is handled similarly. Suppose that $T, T^{\prime} \in \mathscr{T}_{\lambda / \mu}$ and $w(T)<_{B} w\left(T^{\prime}\right)$. If $l\left(w\left(T^{\prime}\right)\right)-l(w(T))=1$ there is no problem, since then $w\left(T^{\prime}\right)$ is obtained from $w(T)$ by a transposition of entries of $T$. However, if $l\left(w\left(T^{\prime}\right)\right)-l(w(T))>1$ we must be sure that we can find an unrefinable chain in Bruhat order from $w(T)$ to $w\left(T^{\prime}\right)$ which consists entirely of images of standard tableaux. Since $w\left(\mathscr{T}_{\lambda / \mu}\right)$ is a left interval, by Corollary 4.6 it is isomorphic, under Bruhat order, to a generalized quotient. Hence by Theorem 3.5, it contains an unrefinable chain in Bruhat order from $w(T)$ to $w\left(T^{\prime}\right)$. This implies that there is a chain of standard tableaux in Bruhat order from $T$ to $T^{\prime}$, which means that $T<_{B} T^{\prime}$.

8. Forest quotients in the symmetric group. We now consider another class of generalized quotients in $\mathscr{S}_{n}$, which arises combinatorially and shares some interesting properties with ordinary quotients. Let $\varphi$ be a planar forest with $n$ nodes, i.e., an ordered collection of planar trees, where a planar tree consists of a root and an ordered collection of planar subtrees. We can picture the forest drawn in the standard way with roots on top and the ordered collections of subtrees drawn from left to right. This allows us to say that node $i$ is to the left of node $j$ if there is a subtree containing node $i$ which precedes a subtree containing node $j$. A standard labeling of $\varphi$ is defined to be a labeling of $\varphi$ with integers, each integer $1,2, \ldots, n$ appearing exactly once, such that the label of a node is less than the label of its parent. Let $\mathscr{F}_{\varphi}$ be the set of all standard labelings of forest $\varphi$. Bruhat order on $\mathscr{F}_{\varphi}$ is defined to be the order relation generated by $F<F^{\prime}$ if $F^{\prime}=(i, j) F$, where the transposition $(i, j)$ acts on labels $i$ and $j$ in $F$ and $i$ appears to the left of $j$ in $F$ and $i<j$. Left order is defined similarly except that $j=i+1$.

There are two well-known linear orderings of a planar forest, and three in the case of binary trees. 
DEFINITION. Let $\varphi$ be a planar forest with trees $\varphi_{1}, \varphi_{2}, \ldots, \varphi_{k}$ ordered from left to right. Postorder is a linear ordering of the nodes of $\varphi$ which is defined recursively as follows:

(1) For $k>1$, first order the nodes of $\varphi_{1}$ in postorder and follow this with the nodes of $\varphi_{2}$ in postorder. Continue ordering the nodes this way, finally putting the postordered nodes of $\varphi_{k}$ last.

(2) For $k=1$, first postorder the subforest obtained by removing the root of $\varphi=\varphi_{1}$ and then let the root come last in the ordering.

Preorder is defined similarly, except that in preorder the root comes before the preordered subforest.

A binary tree is a planar tree which is either empty or consists of a root and left and right subtrees (which can be empty). For binary trees, inorder is a linear ordering of the nodes defined by a similar recursive scheme except that the root comes between the inordered left and right subtrees.

To illustrate these definitions, consider the tree

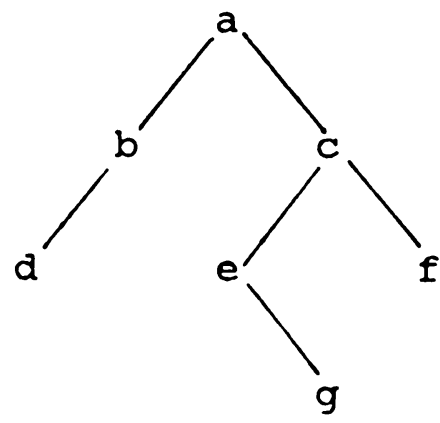

Here, postorder $=$ dbge $f c a$, preorder $=\operatorname{abdceg} f$, and inorder $=d b a e g c f$.

We will say that a linear ordering of the nodes of a planar forest $\varphi$ is recursive if the nodes of each subtree of $\varphi$ occur as a consecutive segment of the ordering. The reason for this name is that such orderings are precisely the ones that can be obtained by the following recursive procedure: Let $\varphi_{1}, \varphi_{2}, \ldots, \varphi_{k}$ be the trees of $\varphi$.

(1) For $k>1$, first order the nodes of each $\varphi_{i}$ recursively. Then concatenate these orderings in any order.

(2) For $k=1$, first recursively order the subforest obtained by removing the root of $\varphi=\varphi_{1}$, and then insert the root after any of the trees, or before the first tree, in the recursively ordered subforest.

In particular, postorder, preorder, and inorder are all recursive orderings.

THEOREM 8.1. Let $\varphi$ be any planar forest.

(1) Both Bruhat order and left order on $\mathscr{F}_{\varphi}$ are graded posets, whose minimum element is the postorder labeling, and whose maximum element is the right to left postorder labeling.

(2) Left order on $\mathscr{F}_{\varphi}$ is a lattice.

(3) Bruhat order on $\mathscr{F}_{\varphi}$ is CL-shellable. 
PROOF. Just as in the proof of Theorem 7.1, we shall provide an isomorphism under Bruhat order and left order from $\mathscr{F}_{\varphi}$ to a generalized quotient in $\mathscr{S}_{n}$. The isomorphism is given in the next theorem.

THEOREM 8.2. Let $\varphi$ be a planar forest with $n$ nodes, and let $w: \mathscr{F}_{\varphi} \rightarrow \mathscr{S}_{n}$ be the map in which $w(F)$ is the permutation obtained by reading the labels of labeled forest $F$ in a fixed order which is either postorder, preorder, or any other recursive ordering. Then $w$ is an isomorphism, under Bruhat order and left order, between $\mathscr{F}_{\varphi}$ and a left interval of $\mathscr{S}_{n}$. Moreover the left interval is a lower left interval when the reading order is postorder.

PROOF. We can think of $\varphi$ as the Hasse diagram of a poset and of $\mathscr{F}_{\varphi}$ as the collection of standard labelings of the poset. One can easily check that any recursive ordering yields a reading order which satisfies condition $(*)$ of Theorem 7.6. Hence, $w\left(\mathscr{F}_{\varphi}\right)$ for these reading orders are left intervals. The rest of the proof is exactly like that of Theorem 7.5.

The map in Theorem 8.2 for which the reading order is postorder shall be called the postorder map, and the preorder map and inorder map are defined similarly.

COROLlaRY 8.3. Let $B T(n)$ be the set of binary trees with $n$ nodes and let $w$ be the inorder map on binary trees. Then the sets $w\left(\mathscr{F}_{\varphi}\right), \varphi \in B T(n)$, partition $\mathscr{S}_{n}$ into $\left(\begin{array}{c}2 n \\ n\end{array}\right) /(n+1)$ disjoint left intervals.

PROOF. For every permutation $\sigma \in \mathscr{S}_{n}$, there is a unique standard-labeled binary tree $T$ such that $w(T)=\sigma$. The label of the root of $T$ is $n$, and its left and right subtrees $T_{1}$ and $T_{2}$ are the unique standard-labeled binary trees satisfying $w\left(T_{1}\right)=\sigma_{1}$ and $w\left(T_{2}\right)=\sigma_{2}$, where $\sigma=\sigma_{1} n \sigma_{2}$. The result now follows from Theorem 8.2 and the well-known fact that $\left(\begin{array}{c}2 n \\ n\end{array}\right) /(n+1)$ is the number of binary trees with $n$ nodes.

We define a forest quotient to be the postorder map image of $\mathscr{F}_{\varphi}$ where $\varphi$ is any planar forest. Note that the ordinary quotients are precisely those forest quotients in which the forest consists entirely of linear trees. An important property of ordinary quotients $W^{J}$, that does not as a rule extend to generalized quotients (e.g., not to all tableau quotients in $\mathscr{S}_{n}$ ), is that every $w \in W$ decomposes uniquely into $u \cdot v$ where $u \in W^{J}$ and $v \in W_{J}$. We say that a generalized quotient $U$ splits $W$, if there exists a set $V \subseteq W$ such that $U=W / V$, and the map $\gamma: U \times V \rightarrow W$ defined by $\gamma(u, v)=u v$ is bijective. Hence, ordinary quotients split $W$. It turns out that forest quotients in $W=\mathscr{S}_{n}$ also split $W$.

Let $x_{1}, x_{2}, \ldots, x_{n}$ be the nodes of the forest $\varphi$ in postorder. For each $i=$ $1,2, \ldots, n$, let $h_{i}(\varphi)$ be the number of nodes of the subtree rooted at $x_{i}$. Note that these nodes are consecutive in postorder. That is, the nodes of the subtree rooted at $x_{i}$ are $x_{j}, x_{j+1}, \ldots, x_{i}$ where $j=i-h_{i}(\varphi)+1$. Let $v_{i}(\varphi)$ be the permutation $s_{i-1} s_{i-2} \cdots s_{j}$, where $s_{\nu}$ is the adjacent transposition $(\nu, \nu+1)\left(s_{\nu}\right.$ acts on the right of a permutation by transposing adjacent symbols). Note that the right interval $\left[e, v_{i}(\varphi)\right]_{R}$ consists only of a single maximal chain

$$
e<_{R} s_{i-1}<_{R} s_{i-1} s_{i-2}<_{R} \cdots<_{R} s_{i-1} s_{i-2} \cdots s_{j}=v_{i}(\varphi) .
$$

THEOREM 8.4. Let $w: \mathscr{F}_{\varphi} \rightarrow \mathscr{S}_{n}$ be the postorder map.

(1) The map

$$
\gamma: w\left(\mathscr{F}_{\varphi}\right) \times\left[e, v_{1}(\varphi)\right]_{R} \times\left[e, v_{2}(\varphi)\right]_{R} \times \cdots \times\left[e, v_{n}(\varphi)\right]_{R} \rightarrow \mathscr{S}_{n},
$$

defined by $\gamma\left(u, w_{1}, w_{2}, \ldots, w_{n}\right)=u w_{1} u_{2} \cdots w_{n}$, is bijective. 
(2) If $u \in w\left(\mathscr{F}_{\varphi}\right)$ and $w_{i} \in\left[e, v_{i}(\varphi)\right]_{R}$ for $i=1,2, \ldots, n$, then

$$
l\left(u w_{1} w_{2} \cdots w_{n}\right)=l(u)+l\left(w_{1}\right)+l\left(w_{2}\right)+\cdots+l\left(w_{n}\right)
$$

(3) The generalized quotient $\mathscr{S}_{n} /\left(\left[e, v_{1}(\varphi)\right]_{R} \cdot\left[e, v_{2}(\varphi)\right]_{R} \cdots\left[e, v_{n}(\varphi)\right]_{R}\right)$ is the same as the forest quotient $w\left(\mathscr{F}_{\varphi}\right)$.

(4) $\left[e, v_{1}(\varphi)\right]_{R} \cdot\left[e, v_{2}(\varphi)\right]_{R} \cdots\left[e, v_{n}(\varphi)\right]_{R}=\left[e, v_{1}(\varphi) v_{2}(\varphi) \cdots v_{n}(\varphi)\right]_{R}$.

(5) The permutation $v_{1}(\varphi) v_{2}(\varphi) \cdots v_{n}(\varphi)$ is the same as the permutation obtained by labeling $\varphi$ in postorder and reading the labels in preorder.

PROOF. If $\varphi$ is the forest with all single node trees, then (1)-(5) hold trivially. Suppose then that some node of $\varphi$ is a parent. Let $m=\min \{j=1,2, \ldots, n \mid$ $x_{j}$ is a parent $\}$. Let $\varphi^{\prime}$ be the forest obtained from $\varphi$ by having all the children of $x_{m}$ in $\varphi$ be siblings of $x_{m}$ in $\varphi^{\prime}$. For example, if

$$
\varphi=0
$$

then

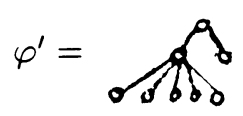

Note that $x_{m}$ has no grandchildren in $\varphi$ since it was chosen to be minimal. We have that $h_{i}(\varphi)=h_{i}\left(\varphi^{\prime}\right)$ if $i \neq m$ and $h_{m}(\varphi)>h_{m}\left(\varphi^{\prime}\right)=1$. Hence, $v_{i}(\varphi)=v_{i}\left(\varphi^{\prime}\right)$ if $i \neq m$ and $v_{m}\left(\varphi^{\prime}\right)=e$. It is also true that $v_{i}(\varphi)=v_{i}\left(\varphi^{\prime}\right)=e$ for $i<m$. It follows that (1) and (2) are consequences of the following lemma by induction on $\sum_{i=1}^{n} h_{i}(\varphi)$.

LEMMA 8.5. (a) The map $\psi: w\left(\mathscr{F}_{\varphi}\right) \times\left[e, v_{m}(\varphi)\right]_{R} \rightarrow w\left(\mathscr{F}_{\varphi^{\prime}}\right)$, defined by $\psi(u, v)$ $=u v$, is a bijection.

(b) For all $u \in w\left(\mathscr{F}_{\varphi}\right)$ and $v \in\left[e, v_{m}(\varphi)\right]_{R}, l(u v)=l(u)+l(v)$.

PROOF. (a) We shall let the permutations in $\mathscr{S}_{n}$ act on labeled forests as follows: If $F$ is a labeling of $\varphi$ and $v \in \mathscr{S}_{n}$, then $F v$ is the labeling of $\varphi$ defined by

$$
F v\left(x_{i}\right)=F\left(x_{v(i)}\right), \quad i=1,2, \ldots, n
$$

Note that $w(F v)=w(F) v$. Hence, (a) is equivalent to showing that the map $\tilde{\psi}: \mathscr{F}_{\varphi} \times\left[e, v_{m}(\varphi)\right]_{R} \rightarrow \mathscr{F}_{\varphi^{\prime}}$ defined by $\tilde{\psi}(F, v)=F v$ is a bijection.

Note that $F \in \mathscr{F}_{\varphi}$ if and only if $F \in \mathscr{F}_{\varphi^{\prime}}$ and $F\left(x_{m}\right)>F\left(x_{i}\right)$ for all $i=$ $j, j+1, \ldots, m-1$ where $j=m-h_{m}(\varphi)+1$. First we show that if $F \in \mathscr{F}_{\varphi}$ and $v \in\left[e, v_{m}(\varphi)\right]_{R}$ then $F v \in \mathscr{F}_{\varphi^{\prime}}$. Let $v=s_{m-1} s_{m-2} \cdots s_{k}$ where $k \geq m-h(m)+1$. To get $F v$ from $F$, the labels of $x_{m}$ and of some of its descendants are cyclically permuted. Note that by the choice of $m$, the descendants are actually children of $x_{m}$ in $\varphi$. Consequently, they are siblings of $x_{m}$ in $\varphi^{\prime}$. Hence $F v$ is obtained from 
$F$ by permuting the labels of childless siblings in $\varphi^{\prime}$. Since $F$ is a standard labeling of $\varphi^{\prime}$, it follows that $F v$ is a standard labeling of $\varphi^{\prime}$.

Now let $F^{\prime}$ be a standard labeling of $\varphi^{\prime}$. Let $k$ be such that $m-h_{m}(\varphi)+1 \leq$ $k \leq m$ and $F^{\prime}\left(x_{k}\right) \geq F^{\prime}\left(x_{i}\right)$ for all $i$ such that $m-h_{m}(\varphi)+1 \leq i \leq m$. In other words, $x_{k}$ has the largest label in the subtree of $\varphi$ rooted at $x_{m}$. Clearly, $v=s_{m-1} s_{m-2} \cdots s_{k}$ is the unique element of $\left[e, v_{m}(\varphi)\right]_{R}$ such that

$$
F^{\prime} v^{-1}\left(x_{m}\right)>F^{\prime} v^{-1}\left(x_{i}\right) \text {, }
$$

for all $i=m-1, m-2, \ldots, m-h_{m}(\varphi)+1$. We have that $F^{\prime} v^{-1}$ is a standard labeling of $\varphi^{\prime}$, since it is obtained from $F^{\prime}$ by permuting the labels of childless siblings in $\varphi^{\prime}$. Therefore by (8.1) we have that $F^{\prime} v^{-1}$ is also a standard labeling of $\varphi$. Since $v$ was uniquely determined, it follows that $\tilde{\psi}$ is a bijection.

(b) Let $u=w(F)$, where $F \in \mathscr{F}_{\varphi}$, and let $v=s_{m-1} s_{m-2} \cdots s_{k}$, where $m-$ $h_{m}(\varphi)+1 \leq k \leq m$. Then $u v=w\left(F s_{m-1} s_{m-2} \cdots s_{k}\right)$. Note that $s_{m-1} s_{m-2} \cdots s_{k}$ acts on $F$ by repeatedly transposing label $F\left(x_{m}\right)$ with labels of children of $x_{m}$. Since these labels are all smaller than $F\left(x_{m}\right)$, each transposition increases the length of the corresponding permutation. Hence $l(u v)=l(u)+m-k=l(u)+l(v)$.

CONTINUATION OF PROOF OF THEOREM 8.4. Parts (1) and (2) have now been proven and we continue with (3) and (4). We will leave it to the reader to check part (5).

(3) It follows from (2) that

$$
w\left(\mathscr{F}_{\varphi}\right) \subseteq \mathscr{S}_{n} /\left(\left[e, v_{1}(\varphi)\right]_{R} \cdot\left[e, v_{2}(\varphi)\right]_{R} \cdots\left[e, v_{n}(\varphi)\right]_{R}\right) .
$$

We now claim that

$$
w\left(\mathscr{F}_{\varphi}\right)=\mathscr{S}_{n} /\left\{v_{1}(\varphi), v_{2}(\varphi), \ldots, v_{n}(\varphi)\right\}
$$

Indeed, $l\left(w v_{i}(\varphi)\right)=l(w)+l\left(v_{i}(\varphi)\right)$ means that $w(i)>w(i-1), w(i-2), \ldots, w(j)$, where $j=i-h_{i}(\varphi)+1$. Since this is precisely the condition that characterizes permutations in $w\left(\mathscr{F}_{\varphi}\right),(8.2)$ holds. It follows that

$$
\begin{aligned}
w\left(\mathscr{F}_{\varphi}\right) & \subseteq \mathscr{S}_{n} /\left(\left[e, v_{1}(\varphi)\right]_{R} \cdot\left[e, v_{2}(\varphi)\right]_{R} \cdots\left[e, v_{n}(\varphi)\right]_{R}\right) \\
& \subseteq \mathscr{S}_{n} /\left\{v_{1}(\varphi), v_{2}(\varphi), \ldots, v_{n}(\varphi)\right\} \\
& =w\left(\mathscr{F}_{\varphi}\right) .
\end{aligned}
$$

We may now conclude that (3) holds.

(4) Let $u$ be the maximum element of $w\left(\mathscr{F}_{\varphi}\right)$. By (1) and (2), the maximum element of $\mathscr{S}_{n}$ equals $u v_{1}(\varphi) v_{2}(\varphi) \cdots v_{n}(\varphi) .\left[e, v_{1}(\varphi) v_{2}(\varphi) \cdots v_{n}(\varphi)\right]_{R}$ is the right generalized quotient $w\left(\mathscr{F}_{\varphi}\right) \backslash \mathscr{S}_{n}$ by Theorem 4.4. But by (2), we have that

$$
\left[e, v_{1}(\varphi)\right]_{R} \cdot\left[e, v_{2}(\varphi)\right]_{R} \cdots\left[e, v_{n}(\varphi)\right]_{R} \subseteq w\left(\mathscr{F}_{\varphi}\right) \backslash \mathscr{S}_{n}
$$

It follows that

$$
\left[e, v_{1}(\varphi)\right]_{R} \cdot\left[e, v_{2}(\varphi)\right]_{R} \cdots\left[e, v_{n}(\varphi)\right]_{R} \subseteq\left[e, v_{1}(\varphi) v_{2}(\varphi) \cdots v_{n}(\varphi)\right]_{R}
$$

For the reverse inclusion it suffices to show that $V=\left[e, v_{1}(\varphi)\right]_{R} \cdot\left[e, v_{2}(\varphi)\right]_{R}$ $\cdots\left[e, v_{n}(\varphi)\right]_{R}$ is a right order ideal in $\mathscr{S}_{n}$. We shall show that if $w_{i} \in\left[e, v_{i}(\varphi)\right]_{R}$ for all $i=1,2, \ldots, n$ and $w_{1} w_{2} \cdots w_{n} s<_{R} w_{1} w_{2} \cdots w_{n}$, where $s \in S$, then $w_{1} w_{2} \cdots w_{n} s \in V$. The following lemma and the strong exchange property imply this. 
LEMMA 8.6. Let $k \leq n$ and $w_{i} \in\left[e, v_{i}(\varphi)\right]_{R}, i=1,2, \ldots, k$. If $w_{1} w_{2} \cdots w_{k} s=$ $w_{1} \cdots \widehat{w}_{m} \cdots w_{k}$ where $1 \leq m \leq k$ and $\widehat{w}_{m}<_{B} w_{m}$, then $\widehat{w}_{m}<_{R} w_{m}$.

ProOF. We prove this by induction on $k$. If $k=1$ or $k=m$ the lemma is trivial. Suppose $m<k$. It follows from (2), the strong exchange property, and the fact that each $w_{i}$ has a unique reduced expression, that $m$ is uniquely determined by $s$ and that $w_{1} w_{2} \cdots w_{k} s<_{R} w_{1} w_{2} \cdots w_{k}$. Since $m<k$, we can conclude that $w_{k} s>_{R} w_{k}$. Since $w_{k}<_{R} v_{k}(\varphi), w_{k}=s_{k-1} s_{k-2} \cdots s_{j}$ where $j \geq k-h_{k}(\varphi)+1$. It follows that $s \neq s_{j}$.

We now show that $s \neq s_{j-1}$. Note that each $w_{i}$ permutes only the numbers, $1,2, \ldots, i$. Consequently, the permutation $w_{1} w_{2} \cdots w_{k-1}$ permutes only the symbols $1,2, \ldots, k-1$ and fixes the symbols $k, k+1, \ldots, n$. It follows from this that $k$ appears in position $j$ in $w_{1} w_{2} \cdots w_{k}$, and the symbols $k+1, k+2, \ldots, n$ are fixed in positions $k+1, k+2, \ldots, n$, respectively. This implies that $w_{1} w_{2} \cdots w_{k} s_{j-1}>_{R}$ $w_{1} w_{2} \cdots w_{k}$. Since $w_{1} w_{2} \cdots w_{k} s<_{R} w_{1} w_{2} \cdots w_{k}$, we may conclude that $s \neq s_{j-1}$. There are now two cases to consider.

Case 1. Suppose $s=s_{i}$, where $i<j-1$. Since $s_{i}$ commutes with each of $s_{k-1}, s_{k-2}, \ldots, s_{j}$, we have

$$
w_{1} \cdots \widehat{w}_{m} \cdots w_{k}=w_{1} w_{2} \cdots w_{k} s=w_{1} w_{2} \cdots w_{k-1} s w_{k} .
$$

This implies that $w_{1} w_{2} \cdots w_{k-1} s=w_{1} \cdots \widehat{w}_{m} \cdots w_{k-1}$. It follows by induction that $\widehat{w}_{m}<_{R} w_{m}$.

Case 2. Suppose $s=s_{i}$, where $i>j$. This time we have that $s_{k-1} s_{k-2} \cdots s_{j} s_{i}=$ $s_{i-1} s_{k-1} s_{k-2} \cdots s_{j}$, since clearly $i<k$. This allows us to conclude that

$$
w_{1} w_{2} \cdots w_{k-1} s_{i-1}=w_{1} \cdots \widehat{w}_{m} \cdots w_{k-1} .
$$

Again by induction we have that $\widehat{w}_{m}<_{R} w_{m}$.

We now consider the dual situation. Let $\mathscr{F}_{\varphi}^{*}$ be the set of dual standard labelings of a planar forest $\varphi$ with $n$ nodes, where a dual standard labeling of $\varphi$ is a labeling with distinct integers $1,2, \ldots, n$, in which the label of a node is greater than that of its parent. Theorems 8.1 and 8.2 hold for $\mathscr{F}_{\varphi}^{*}$ with preorder replacing postorder. Theorem 8.4, appropriately modified, also remains true for $\mathscr{F}_{\varphi}^{*}$. We summarize this in the following theorem.

THEOREM 8.7. Let $\varphi$ be a planar forest with $n$ nodes, let $w: \mathscr{F}_{\varphi} \rightarrow \mathscr{S}_{n}$ be the postorder map, and let $w^{*}: \mathscr{F}_{\varphi}^{*} \rightarrow \mathscr{S}_{n}$ be the preorder map. Then the following hold:

(1) $w\left(\mathscr{F}_{\varphi}\right)$ is the generalized quotient $\mathscr{S}_{n} /[e, v]_{R}$, where $v$ is the permutation obtained by labeling $\varphi$ in postorder and reading the labels in preorder.

(2) $w\left(\mathscr{F}_{\varphi}\right)$ is the interval $[e, u]_{L}$, where $u$ is the permutation obtained by labeling $\varphi$ in right to left postorder and reading the labels in postorder.

(3) $w^{*}\left(\mathscr{F}_{\varphi}^{*}\right)$ is the generalized quotient $\mathscr{S}_{n} /\left[e, v^{-1}\right]_{R}$, where $v$ is as in (1), i.e., $v^{-1}$ is obtained by labeling $\varphi$ in preorder and reading the labels in postorder.

(4) $w^{*}\left(\mathscr{F}_{\varphi}^{*}\right)$ is the interval $\left[e, u^{*}\right]_{L}$, where $u^{*}$ is obtained by labeling $\varphi$ in right to left preorder and reading the labels in preorder.

(5) $[e, v]_{L},\left[e, v^{-1}\right]_{L},[e, u]_{L},\left[e, u^{-1}\right]_{L},\left[e, u^{*}\right]_{L}$, and $\left[e, u^{*-1}\right]_{L}$ are all splitting quotients in $\mathscr{P}_{n}$.

Note that if $\varphi$ is a forest of all linear trees, then Theorem 8.7 reduces to the fact that $W_{J}$ and $W^{J}$ are splitting quotients of $\mathscr{P}_{n}$. It would be interesting to find a characterization of those permutations $w$ for which $[e, w]_{L}$ splits $\mathscr{S}_{n}$. 
9. Combinatorial structure of some quotients in the symmetric group. The ordinary quotients $W^{J}$ of the symmetric group $W=\mathscr{S}_{n}$ have been generalized in two directions in the preceding sections. On the one hand, $W^{J}$ is a special case of a descent class, and descent classes are special cases of tableau quotients. On the other hand, $W^{J}$ is a special case of a forest quotient, namely for a forest with linear trees. In this section we will show that a number of combinatorial properties known for ordinary quotients $W^{J}$ in the symmetric group extend to these larger classes of quotients.

For any subset $A$ of a Coxeter group $W$, let $A(q)=\sum_{w \in A} q^{l(w)}$. If $A$ is a graded poset under Bruhat order or weak order, then $A(q)$ is the rank generating function of $A$, up to a power of $q$. For each integer $k \geq 1$, let $[k]=1+q+q^{2}+\cdots+q^{k-1}$ and $[k] !=[k] \cdot[k-1] \cdots[1]$.

THEOREM 9.1. Let $\varphi$ be a planar forest with $n$ nodes, and let $A_{\varphi}=w\left(\mathscr{F}_{\varphi}\right)$ or $A_{\varphi}=w^{*}\left(\mathscr{F}_{\varphi}^{*}\right)$, where $w: \mathscr{F}_{\varphi} \rightarrow \mathscr{S}_{n}$ is the postorder map and $w^{*}: \mathscr{F}_{\varphi}^{*} \rightarrow \mathscr{S}_{n}$ is the preorder map. Then,

$$
A_{\varphi}(q)=[n] ! / \prod_{i=1}^{n}\left[h_{i}\right],
$$

where $h_{i}$ is the size of the subtree rooted at the ith node.

(b) $A_{\varphi}(q)$ is a reciprocal polynomial, i.e., in $A_{\varphi}(q)$, the coefficient of $q^{i}$ is equal to the coefficient of $q^{d-i}$, where $d=\operatorname{deg} A_{\varphi}(q)$.

ProOF. (a) We shall prove this for $A_{\varphi}=w\left(\mathscr{F}_{\varphi}\right)$. The proof for $A_{\varphi}=w^{*}\left(\mathscr{F}_{\varphi}^{*}\right)$ is similar.

By (1) and (2) of Theorem 8.4 we have

$$
\begin{aligned}
\mathscr{S}_{n}(q) & =\sum_{\substack{u \in w\left(\mathscr{F}_{\varphi}\right) \\
w_{i} \in\left[e, v_{i}(\varphi)\right]_{R}}} q^{l\left(u w_{1} w_{2} \cdots w_{n}\right)} \\
& =A_{\varphi}(q) \cdot \prod_{i=1}^{n}\left(\sum_{w_{i} \in\left[e, v_{i}(\varphi)\right]_{R}} q^{l\left(w_{i}\right)}\right) .
\end{aligned}
$$

It is well known that $\mathscr{S}_{n}(q)=[n] !($ cf. $[\mathbf{1}])$. Since $\left[e, v_{i}(\varphi)\right]_{R}$ is a single unrefinable chain of cardinality $h_{i}$, we also have

$$
\sum_{w_{i} \in\left[e, v_{i}(\varphi)\right]_{R}} q^{l\left(w_{i}\right)}=1+q+\cdots+q^{h} i^{-1}=\left[h_{i}\right] .
$$

Hence,

$$
[n] !=A_{\varphi}(q) \cdot \prod_{i=1}^{n}\left[h_{i}\right]
$$

(b) By Theorem 8.2,w(F्F $)$ and $w^{*}\left(\mathscr{F}_{\varphi}\right)$ are isomorphic under Bruhat order and left order. Let $\sigma: w^{*}\left(\mathscr{F}_{\varphi}\right) \rightarrow w^{*}\left(\mathscr{F}_{\varphi}^{*}\right)$ be the map defined by $\sigma(w)=w_{0} w$, where $w_{0}$ is the maximum permutation of $\mathscr{S}_{n}$, i.e.,: $n, n-1, \ldots, 1$. Recall that multiplication by $w_{0}$ on the left of a permutation serves to interchange the symbols 1 and $n, 2$ and $n-1$, etc. Hence, $u \in w^{*}\left(\mathscr{F}_{\varphi}\right)$ if and only if $\sigma(u) \in w^{*}\left(\mathscr{F}_{\varphi}^{*}\right)$. Therefore $\sigma$ is a bijection. It is also clear that $\sigma$ and its inverse are order reversing. Combining this 
with the fact that $w\left(\mathscr{F}_{\varphi}\right)$ and $w^{*}\left(\mathscr{F}_{\varphi}\right)$ are isomorphic allows us to conclude that $w^{*}\left(\mathscr{F}_{\varphi}^{*}\right)$ is isomorphic to the dual of $w\left(\mathscr{F}_{\varphi}\right)$. Since by part (a) the rank generating function of $w\left(\mathscr{F}_{\varphi}\right)$ is equal to that of $w^{*}\left(\mathscr{F}_{\varphi}^{*}\right)$, we have that the rank generating function of $w\left(\mathscr{F}_{\varphi}\right)$ is equal to that of its dual.

Part (a) of Theorem 9.1 is a $q$-analogue of Knuth's [17, p. 70] hook-length formula for the number of standard labelings of a planar forest. Another $q$-analogue of the hook-length formula is given by Stanley in [19]. His formula is identical to ours except that instead of $q$-counting in terms of the number of inversions (i.e., length) of the permutation, he uses the major index of the inverse of the permutation. By combining our result with Stanley's we obtain the curious fact that $q$-counting forest quotients in terms of inversion numbers is the same as $q$-counting in terms of inverse major index. In [11] we use a bijection of Foata to prove the identity directly, and in $[\mathbf{1 0}]$ we show that this identity can actually be used to characterize forest quotients.

When $\varphi$ is a forest with linear trees, part (a) of Theorem 9.1 reduces to the formula expressing the rank generating function of an ordinary quotient in $\mathscr{S}_{n}$ as a $q$-multinomial coefficient. It is easy to see that this formula is actually equivalent to MacMahon's well-known formula expressing the distribution of the number of inversions of multiset permutations as a $q$-multinomial coefficient (see $[\mathbf{1}$, p. 41]).

In connection with part (b) of Theorem 9.1 we remark that forest quotients $A_{\varphi}$ are not necessarily isomorphic to their duals as posets. E.g., this fails for both Bruhat and left order for the tree

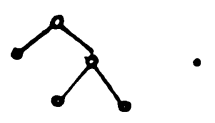

However, for forests with left-right symmetry the maximal element in the quotient is an involution, and hence isomorphism with the dual follows from Theorem 3.10.

In [11] we show that the forest quotients are not only rank symmetric but also rank unimodal.

A stronger property of ranked posets than that of being rank symmetric is the symmetric chain decomposition property. This means that $P$ can be partitioned into disjoint unrefinable chains $x_{i}<x_{i+1}<\cdots<x_{r-i}$, where $r$ is the length of $P$ and the rank of $x_{j}$ is $j$ for $i \leq j \leq r-i$. It is known that all parabolic subgroups of $\mathscr{S}_{n}$ (often known as Young subgroups) have the symmetric chain decomposition property under Bruhat order [21, p. 182]. This result generalizes to other left intervals of $\mathscr{P}_{n}$.

THEOREM 9.2. Let $\varphi$ be any planar forest with $n$ nodes. If $v$ is the permutation obtained by labeling $\varphi$ in postorder and reading the labels in preorder or vice versa, then $[e, v]_{L}$ has the symmetric chain decomposition property under Bruhat order.

PROOF. Let $v$ be the permutation obtained by labeling $\varphi$ in postorder and reading the labels in preorder. Then $v^{-1}$ is the permutation obtained by labeling $\varphi$ in preorder and reading the labels in postorder. By Theorem 8.4, the map

$$
\psi:\left[e, v_{1}(\varphi)\right]_{R} \times\left[e, v_{2}(\varphi)\right]_{R} \times \cdots \times\left[e, v_{n}(\varphi)\right]_{R} \rightarrow[e, v]_{R},
$$


defined by $\psi\left(w_{1}, w_{2}, \ldots, w_{n}\right)=w_{1} w_{2} \cdots w_{n}$, is bijective. The map induces a partial order relation on $[e, v]_{R}$, which is isomorphic to the direct product of right order on the intervals $\left[e, v_{i}(\varphi)\right]_{R}$. By $(2)$ of Theorem 8.4, this order is weaker than Bruhat order on $[e, v]_{R}$ but it has the same rank function. Recall that each interval $\left[e, v_{i}(\varphi)\right]_{R}$ is simply a chain under right order. The induced order on $[e, v]_{R}$ is hence isomorphic to a product of chains. It is well known that a product of chains has the symmetric chain decomposition property (cf. [21, p. 182]). Clearly, any partial order that is stronger than, and has the same rank function as, a partial order with the symmetric chain decomposition property, also has the symmetric chain decomposition property. Hence, $[e, v]_{R}$ has the symmetric chain decomposition property under Bruhat order. By left-right symmetry, $\left[e, v^{-1}\right]_{L}$ also has the symmetric chain decomposition property under Bruhat order. The same argument, using the version of Theorem 8.4 for dual standard labelings and the preorder map, implies that $\left[e, v^{-1}\right]_{R}$ and $[e, v]_{L}$ have the symmetric chain decomposition property under Bruhat order.

We now turn to tableau quotients. A general formula for $q$-counting tableau quotients does unfortunately not seem to exist. However, for the special case of descent classes $D_{I}^{J}$ there is the following determinantal formula. For the case $I=\varnothing$, this again specializes to the multinomial formula for $W^{\bar{J}}(q)$. For the case $I=J$, the formula was previously obtained by Stanley [20], as a $q$-analogue to a result of MacMahon (viz., the $I=J$ and $q=1$ case).

THEOREM 9.3. Let $S=\left\{s_{1}, s_{2}, \ldots, s_{n-1}\right\}$ be the set of adjacent transpositions $s_{i}=(i, i+1)$ in $\mathscr{S}_{n}$. Suppose $I \subseteq J=\left\{s_{i_{1}}, s_{i_{2}}, \ldots, s_{i_{j}}\right\} \subseteq S, 1 \leq i_{1}<i_{2}<\cdots<$ $i_{j} \leq n-1$. Then

$$
D_{I}^{J}(q)=[n] ! \operatorname{det}\left(\begin{array}{ccccc}
\frac{1}{\left[i_{1}\right] !} & \frac{1}{\left[i_{2}\right] !} & \frac{1}{\left[i_{3}\right] !} & \cdots & \frac{1}{[n] !} \\
* & \frac{1}{\left[i_{2}-i_{1}\right] !} & \frac{1}{\left[i_{3}-i_{1}\right] !} & \cdots & \frac{1}{\left[n-i_{1}\right] !} \\
0 & * & \frac{1}{\left[i_{3}-i_{2}\right] !} & \cdots & \frac{1}{\left[n-i_{2}\right] !} \\
0 & 0 & * & \cdots & \frac{1}{\left[n-i_{3}\right] !} \\
\vdots & \vdots & \vdots & & \vdots \\
0 & 0 & 0 & \cdots & \frac{1}{\left[n-i_{j}\right] !}
\end{array}\right)
$$

where the subdiagonal element * in the pth column equals one if $s_{i_{p}} \in I$, and is zero otherwise, $1 \leq p \leq j$.

ProOF. First let $(W, S)$ be any Coxeter group, and $I \subseteq J \subseteq S$. We shall prove the formula

$$
D_{I}^{J}(q)=\sum_{J-I \subseteq K \subseteq J}(-1)^{|J-K|} \frac{W(q)}{W_{\bar{K}}(q)},
$$

where $\bar{K}=S-K$ (cf. [12, p. 45]).

Since $W^{\bar{K}}=D_{\varnothing}^{K}$, and hence

$$
\frac{W(q)}{W_{\bar{K}}(q)}=W^{\bar{K}}(q)=\sum_{L \subseteq K} D_{L}(q),
$$


we get for the right-hand side of (9.1)

$$
\begin{aligned}
\mathrm{RHS} & =\sum_{J-I \subseteq K \subseteq J}(-1)^{|J-K|} \sum_{L \subseteq K} D_{L}(q) \\
& =\sum_{L \subseteq J} D_{L}(q) \sum_{(J-I) \cup L \subseteq K \subseteq J}(-1)^{|J-K|} \\
& =\sum_{I \subseteq L \subseteq J} D_{L}(q)=D_{I}^{J}(q) .
\end{aligned}
$$

Now, for $W=\mathscr{S}_{n}$ and $K=\left\{s_{e_{1}}, s_{e_{2}}, \ldots, s_{e_{k}}\right\} \subseteq S, 1 \leq e_{1}<e_{2}<\cdots<e_{k} \leq$ $n-1$, we know that $W(q)=[n]$ !, and therefore also that

$$
W_{\bar{K}}(q)=\left[e_{1}\right] !\left[e_{2}-e_{1}\right] ! \cdots\left[n-e_{k}\right] ! .
$$

Hence, (9.1) takes the form

$$
D_{I}^{J}(q)=[n] ! \sum_{J-I \subseteq K \subseteq J}(-1)^{|J-K|} \frac{1}{\left[e_{1}\right] !\left[e_{2}-e_{1}\right] ! \cdots\left[n-e_{k}\right] !} .
$$

It is a simple exercise to see that the summation on the right-hand side can be rewritten in the stated determinantal form.

For the case $I=\left\{s_{2}, s_{4}, s_{6}, \ldots\right\}$, the descent class of all alternating permutations, the polynomials $D_{I}^{I}(q)$ are the $q$-tangent and $q$-secant numbers. See Andrews and Foata [2] for results and further references concerning these.

It is known that tableau quotients for two-row shapes with nonoverlapping rows are distributive lattices under Bruhat order. In fact, such quotients are ordinary quotients $W^{J}$ where $|S-J|=1$, and Bruhat order is identical to left order and is isomorphic to the inclusion ordering of Ferrers diagrams fitting into a rectangle, $\mathrm{cf}$. [21]. We now observe that this generalizes to all two-row shapes.

Suppose $\lambda / \mu$ has two rows, of length $k$ and $l$, respectively, which are overlapping along $m$ boxes:

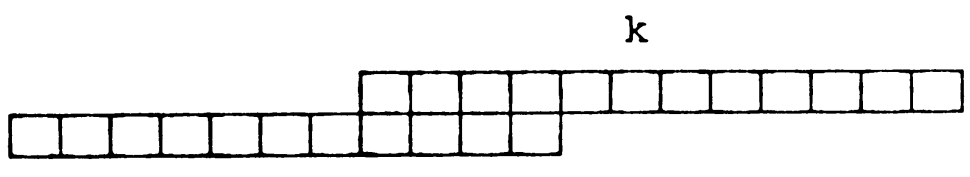

m

$\ell$

Note that if $w\left(\mathscr{T}_{\lambda / \mu}\right)$ is the set of row-order tableau words then $w\left(\mathscr{T}_{\lambda / \mu}\right)$ is a lower left interval of the ordinary quotient $W^{J}$, where $J=S-(k, k+1)$. This implies that Bruhat order on $\mathscr{T}_{\lambda / \mu}$ is identical to left order on $\mathscr{T}_{\lambda / \mu}$ and is a distributive lattice which is isomorphic to the inclusion ordering of Ferrers diagrams fitting into some fixed Ferrers diagram. The isomorphism, which is the restriction of the isomorphism given in [21, p. 173], can be described as follows: For $T \in \mathscr{T}_{\lambda / \mu}$, let $\psi(T)=\left(d_{1}, d_{2}, \ldots, d_{k}\right)$, where $d_{i}$ is the number of entries in the bottom row of $T$ which are less than the $i$ th entry of the top row. In other words, $\psi(T)$ is the essential part of the inversion sequence of the row-order tableau word $w(T)$. It is clear that $0 \leq d_{1} \leq d_{2} \leq \cdots \leq d_{k} \leq l$, and that $d_{i} \leq l-m+i-1$ for $1 \leq i \leq m$. 
In other words, the Ferrers diagram whose row sizes are $d_{k}, d_{k-1}, \ldots, d_{1}$ fits into the staircase-truncated rectangle $R_{k, l}^{m}$ :

$\ell$

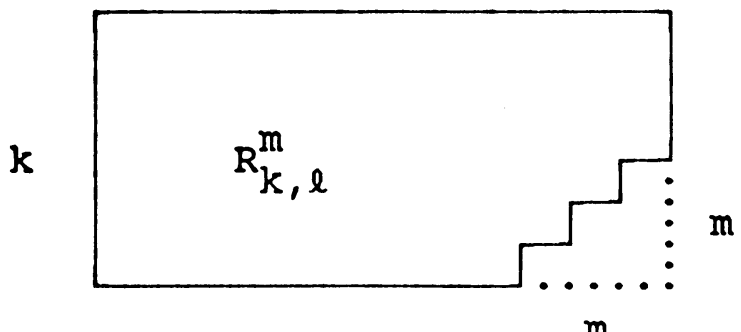

m

Conversely, any Ferrers diagram fitting into $R_{k, l}^{m}$ arises from $\psi(T)$, for a unique $T \in \mathscr{T}_{\lambda / \mu}$. Hence, $\psi$ is a bijection $\mathscr{T}_{\lambda / \mu} \rightarrow\left[\varnothing, R_{k, l}^{m}\right]$, where the latter symbol stands for the interval below $R_{k, l}^{m}$ in the Young lattice of all Ferrers diagrams ordered by inclusion. It is also easy to see that $\psi$ is, in fact, an isomorphism. We now conclude that:

THEOREM 9.4. For a two-row shape $\lambda / \mu$ as above, $\mathscr{T}_{\lambda / \mu}$ under Bruhat order or left order is isomorphic to the poset of Ferrers diagrams fitting into $R_{k, l}^{m}$ ordered by inclusion. In particular, $\mathscr{T}_{\lambda / \mu}$ is a distributive lattice.

We observe that Bruhat order on the tableau quotient $C_{k}$ for the $2 \times k$ rectangular shape (i.e., the case $k=l=m$ ) is isomorphic to the lattice of Ferrers diagrams fitting into the staircase diagram $(k-1, k-2, \ldots, 1)$. Its rank generating function $C_{k}(q)$ is a natural $q$-analogue of the Catalan numbers, which has been studied by L. Carlitz and others, see [15].

10. Appendix: Convexity. In [22] Tits introduced a notion of convexity for Coxeter complexes. This can be translated into terms of left order of Coxeter groups, and in $\S 5$ we used this order-theoretic formulation to conclude that generalized quotients are convex as subsets of the group.

In this appendix, we will give a direct approach to convexity in the ordertheoretic language of this paper. Tits' results will be proven along with some added details.

Recall that a subset $C$ of a Coxeter group $W$ is called convex (under left order) if for all $u, w \in C$, every minimum length path from $u$ to $w$ in the Hasse diagram of the left ordering of $W$ is in $C$. Equivalently, if $u, w \in C$ and $w u^{-1}=s_{1} s_{2} \cdots s_{k}$ is a reduced expression then $s_{j} s_{j+1} \cdots s_{k} u \in C$ for all $1 \leq j \leq k$.

Let $\tilde{T}_{w}=T-T_{w^{-1}}=\left\{t \in T \mid w t>_{B} w\right\}$, for all $w \in W$. For $x_{1}, x_{2}, \ldots, x_{n} \in W$, let $\operatorname{Conv}\left(x_{1}, x_{2}, \ldots, x_{n}\right)$ denote the convex hull of the set $X=\left\{x_{1}, x_{2}, \ldots, x_{n}\right\}$, i.e., the intersection of all convex sets that contain $X$. The following is equivalent to Corollary 2.23 in [22].

$$
\begin{aligned}
& \text { THEOREM 10.1 (TITS). For } x, y \in W \\
& \begin{aligned}
\operatorname{Conv}(x, y)= & \left\{z \in W \mid \tilde{T}_{x} \cap \tilde{T}_{y} \subseteq \tilde{T}_{z} \subseteq \tilde{T}_{x} \cup \tilde{T}_{y}\right\} \\
= & \{z \in W \mid z \text { is on a minimum length path from } x \text { to } y\} .
\end{aligned}
\end{aligned}
$$


ProOF. Let $B$ and $C$ be the second and third sets, respectively. We begin by showing that $B=C$. Suppose that $z$ is on a minimum length path from $x$ to $y$. Note that this is equivalent to saying that $z=u x$ and $z=v y$ for some $u, v \in W$ such that $\left\langle v^{-1}\right\rangle\langle u\rangle$ is reduced. Suppose that $t \in \tilde{T}_{x} \cap \tilde{T}_{y}$. If $z t<_{B} z$ then by Lemma $2.4, z t=\widehat{u} x$ and $z t=\widehat{v} y$, where $\widehat{u}<_{B} u$ and $\widehat{v}<_{B} v$. It follows that $\widehat{u} x=\widehat{v} y$, which implies that $\widehat{v}^{-1} \widehat{u}=y x^{-1}=v^{-1} u$. But this contradicts the fact that $\left\langle v^{-1}\right\rangle\langle u\rangle$ is reduced. Hence, $z t>_{B} z$, or equivalently, $t \in \tilde{T}_{z}$, and therefore, $\tilde{T}_{x} \cap \tilde{T}_{y} \subseteq \tilde{T}_{z}$.

Now suppose that $t \in \tilde{T}_{z}$. If $t \notin \tilde{T}_{x} \cup \tilde{T}_{y}$ then $x t<_{B} x$ and $y t<_{B} y$. This means that $x t t>_{B} x t$ and $y t t>_{B} y t$. It follows that $t \in \tilde{T}_{x t} \cap \tilde{T}_{y t}$. Since $z t=u x t$ and $z t=$ $v y t$, we may repeat the argument in the preceding paragraph, substituting $x t, y t$, and $z t$ for $x, y$, and $z$, respectively, to conclude that $z t t>_{B} z t$. But this contradicts the assumption that $t \in \tilde{T}_{z}$. Hence, $t \in \tilde{T}_{x} \cup \tilde{T}_{y}$ and therefore, $\tilde{T}_{z} \subseteq \tilde{T}_{x} \cup \tilde{T}_{y}$. Combining this with the previous paragraph results in $\tilde{T}_{x} \cap \tilde{T}_{y} \subseteq \tilde{T}_{z} \subseteq \tilde{T}_{x} \cup \tilde{T}_{y}$. Since $z$ was an arbitrary element of $C$, we have $C \subseteq B$.

For the reverse inclusion suppose that $z \in B$. Let $a$ and $b$ be such that $z=a x$ and $y=b x$. We shall show that $a<_{L} b$, which is equivalent to showing that $z$ is on a minimum length path from $x$ to $y$. According to Proposition 2.5, it suffices to show that $\tilde{T}_{b} \subseteq \tilde{T}_{a}$. Suppose $t \notin \tilde{T}_{a}$. Then at $<_{B} a$. Let $t^{\prime}=x^{-1} t x$.

First we claim that $z t^{\prime}>_{B} z$ implies $x t^{\prime}<_{B} x$ and that $z t^{\prime}<_{B} z$ implies $x t^{\prime}>_{B} x$. Let $z t^{\prime}>_{B} z$. We have $z t^{\prime}=a x t^{\prime}=a t x=\widehat{a} x$, where $\widehat{a}<_{B} a$. If $x t^{\prime}>_{B} x$ then by Lemma $2.4, z=z t^{\prime} t^{\prime}=\widehat{a} x t^{\prime}=\bar{a} x$, where $\bar{a}<_{B} \widehat{a}$. It follows that $\bar{a}=a$, which is impossible. Hence, the first implication holds. The second implication follows from the first implication with $z t^{\prime}$ and $x t^{\prime}$ playing the roles of $z$ and $x$, respectively.

There are now two cases to consider.

Case 1. Suppose $z t^{\prime}<_{B} z$. Then by the above claim $x t^{\prime}>_{B} x$. Since $\tilde{T}_{x} \cap \tilde{T}_{y} \subseteq$ $\tilde{T}_{z}$, it follows that $y t^{\prime}<_{B} y$. By Lemma 2.4, we have that $b x t^{\prime}=\widehat{b} x$, where $\widehat{b}<_{B} b$. But we also have that $b x t^{\prime}=b t x$. Hence, $b t=\widehat{b}$, or equivalently $t \notin \tilde{T}_{b}$. We may now conclude that $\tilde{T}_{b} \subseteq \tilde{T}_{a}$, for this case.

Case 2. Suppose $z t^{\prime}>_{B} z$. Again by the above claim, $x t^{\prime}<_{B} x$. Now since $\tilde{T}_{z} \subseteq \tilde{T}_{x} \cup \tilde{T}_{y}$, we have $y t^{\prime}>_{B} y$. Since $x t^{\prime} t^{\prime}>_{B} x t^{\prime}$ and $y t^{\prime} t^{\prime}<_{B} y t^{\prime}$, it follows from Lemma 2.4 that $y=y t^{\prime} t^{\prime}=\widehat{b} x t^{\prime}$, where $\widehat{b}<_{B} b$. But we also have that $y=b x=b t x t^{\prime}$. Hence, $b t=\widehat{b}$, and just as in Case $1, \tilde{T}_{b} \subseteq \tilde{T}_{a}$.

Since $\tilde{T}_{b} \subseteq \tilde{T}_{a}$ holds in both cases, we can conclude that $z$ is on a minimum length path from $x$ to $y$, and therefore that $B \subseteq C$. Combining this with the reverse inclusion gives $B=C$.

We shall now show that $B$ is a convex set. Let $u, v \in B$ and let $C^{\prime}$ be the set of all elements on a minimum length path from $u$ to $v$. We have already shown that if $z \in C^{\prime}$ then $\tilde{T}_{u} \cap \tilde{T}_{v} \subseteq \tilde{T}_{z} \subseteq \tilde{T}_{u} \cup \tilde{T}_{v}$. But since $u, v \in B$, we have $\tilde{T}_{x} \cap \tilde{T}_{y} \subseteq \tilde{T}_{u} \cap \tilde{T}_{v}$ and $\tilde{T}_{u} \cup \tilde{T}_{v} \subseteq \tilde{T}_{x} \cup \tilde{\tilde{T}}_{y}$. It follows that $\tilde{T}_{x} \cap \tilde{T}_{y} \subseteq \tilde{T}_{z} \subseteq \tilde{T}_{x} \cup \tilde{T}_{y}$, which implies that $C^{\prime} \subseteq B$ and hence that $B$ is convex. A consequence of this is that $\operatorname{Conv}(x, y) \subseteq B$. Since the inclusion $C \subseteq \operatorname{Conv}(x, y)$ clearly holds, all three sets are identical.

COROLlaRY 10.2. Every left interval in $W$ is a convex subset.

We define the rank of a convex set $C$ to be the cardinality of a minimum cardinality set $\left\{x_{1}, x_{2}, \ldots, x_{m}\right\}$ such that $C=\operatorname{Conv}\left(x_{1}, x_{2}, \ldots, x_{m}\right)$, if a finite such set exists. Thus $\operatorname{rank}(C)=2$ if and only if $C=\operatorname{Conv}(x, y)$ for some $x \neq y$. For 
$x, y \in W$, let $d(x, y)$ denote the distance between $x$ and $y$, i.e., the length of a minimum length path from $x$ to $y$ in the Hasse diagram. Equivalently, $d(x, y)=l\left(y x^{-1}\right)$. The diameter of a convex set $C$ is defined to be the maximum distance $d(x, y)$ over all $x, y \in C$, if such a maximum exists.

THEOREM 10.3. Let $C \subseteq W$ be a convex set of rank 2 and diameter $n$. Then for any $x, y \in C, C=\operatorname{Conv}(x, y)$ if and only if $d(x, y)=n$.

Proof. $(\Rightarrow)$ Clearly, $d(x, y) \leq \operatorname{diam}(C)=n$. On the other hand, if $a, b \in$ Conv $(x, y)$ then by Theorem 10.1 , there exist minimum length paths from $x$ and $y$ through $a$ and $b$. Let $P_{1}, P_{2}, P_{3}, P_{4}$ be paths such that

$$
x \stackrel{P_{1}}{-} a \stackrel{P_{2}}{-} y \text { and } \quad x \stackrel{P_{3}}{-} b \stackrel{P_{4}}{-} y
$$

are minimum length paths from $x$ to $y$. Then

$$
\operatorname{length}\left(P_{1}\right)+\operatorname{length}\left(P_{2}\right)=\operatorname{length}\left(P_{3}\right)+\operatorname{length}\left(P_{4}\right)=d(x, y) .
$$

But then length $\left(P_{1}\right)+$ length $\left(P_{3}\right) \leq d(x, y)$ or length $\left(P_{2}\right)+\operatorname{length}\left(P_{4}\right) \leq d(x, y)$, which shows that $d(a, b) \leq d(x, y)$. Hence $n=\operatorname{diam}(C) \leq d(x, y)$.

$(\Leftarrow)$ Choose $a, b \in C$ so that $C=\operatorname{Conv}(a, b)$. Then by $(\Rightarrow) d(a, b)=n$. It suffices to show that $a, b$ are on minimum length paths from $x$ to $y$, since then $C=\operatorname{Conv}(a, b) \subseteq \operatorname{Conv}(x, y) \subseteq C$. We know, by Theorem 10.1, that $x, y$ are on minimum length paths from $a$ to $b$, say

$$
a \stackrel{P_{1}}{-} x-\frac{P_{2}}{-} b \quad \text { and } \quad a \stackrel{P_{3}}{-} y \stackrel{P_{4}}{-} b
$$

where

$$
\text { length }\left(P_{1}\right)+\operatorname{length}\left(P_{2}\right)=\operatorname{length}\left(P_{3}\right)+\operatorname{length}\left(P_{4}\right)=n \text {. }
$$

Since $d(x, y)=n$, we also have that length $\left(P_{1}\right)+\operatorname{length}\left(P_{3}\right) \geq n$ and $\operatorname{length}\left(P_{2}\right)+$ length $\left(P_{4}\right) \geq n$. But since the sum of the lengths of the two paths from $x$ to $y$ is $2 n$, both of these paths must have length $n$, which means that both paths are minimum length paths.

We are now ready to prove Theorem 5.3, that is, Tits' characterization of convex sets.

Proof OF ThEOREM 5.3. ( $\Leftarrow)$ Let $x, y \in W_{D}^{A}$. Then $A \subseteq \tilde{T}_{x} \cap \tilde{T}_{y}$ and $\tilde{T}_{x} \cup \tilde{T}_{y} \subseteq T-D$. If $z \in \operatorname{Conv}(x, y)$ then by Theorem 10.1, $A \subseteq \tilde{T}_{z} \subseteq T-D$, which means that $z \in W_{D}^{A}$. It follows that $W_{D}^{A}$ is convex. That $W^{A}$ is an order ideal follows from Proposition 2.5, and also from the convexity since $e \in W^{A}$.

$(\Rightarrow)$ We prove this first for the case that $C$ is an order ideal. Let $M$ be the set of minimal elements of $W-C$ under left order and let

$$
A=\left\{w^{-1} s w \mid w \in M, s \in S, s w<_{L} w\right\} .
$$

We shall prove that $C=W^{A}$.

Let $u \in W^{A}$. Suppose $u \notin C$. Then there is some element $w \in M$ such that $w \leq_{L} u$. Let $s \in S$ be such that $s w<_{L} w$ and let $t=w^{-1} s w$. Clearly, $t \in A$ and $t \notin \tilde{T}_{w}$. Since by Proposition $2.5, \tilde{T}_{u} \subseteq \tilde{T}_{w}$, we have that $t \notin \tilde{T}_{u}$. But this contradicts the fact that $u \in W^{A}$. Hence, $u \in C$, and therefore, $W^{A} \subseteq C$.

Now let $u \in C$. Suppose $u \notin W^{A}$. Then there is some $t \in A$ such that $u t<_{B} u$. Since $t \in A, t=w^{-1} s w$ for some $w \in M$ and $s \in S$, where $s w<_{L} w$. Note that 
$\tilde{T}_{w} \cup\{t\}=\tilde{T}_{s w}$. Since $t \notin \tilde{T}_{u}, \tilde{T}_{u} \cap \tilde{T}_{s w} \subseteq \tilde{T}_{w}$. It is also true that $\tilde{T}_{w} \subseteq \tilde{T}_{u} \cup \tilde{T}_{s w}$. Hence, by Theorem 10.1, $w \in \operatorname{Conv}(u, s w)$. Since $w$ is a minimal element of $W-C$ under left order, $s w \in C$. This leads us to conclude that $w \in C$, which contradicts $w \in M$. Hence, $u \in W^{A}$, and consequently, $C=W^{A}$.

To prove the general case we need the following lemma.

Lemma 10.4. Let $u, v \in W$, and $t \in T$. If $u<_{B}$ ut and $v<_{B}$ tv then $u v<_{B} u t v$.

Proof. Suppose that $u v>_{B} u t v$. Then $u v>_{B} t^{\prime} u v$, where $t^{\prime}=u t u^{-1} \in T$. By Lemma 2.4, $u t v=t^{\prime} u v=u \widehat{v}$ for some $\widehat{v}<_{B} v$. It follows that $t v=\widehat{v}$, which contradicts the assumption that $t v>_{B} v$.

PROOF OF THEOREM 5.3 (CONTINUED). Let $C$ be a general convex subset. For any $x \in C, C \cdot x^{-1}$ is a convex order ideal. It follows from the order ideal case that $C=W^{E} \cdot x$ for some $E \subseteq T$. We will now show that $W^{E} \cdot x=W_{D}^{A}$, where $A=\left\{x^{-1} t x \mid t \in E, t x>_{B} x\right\}$ and $D=\left\{x^{-1} t x \mid t \in E, t x<_{B} x\right\}$.

Let $w \in W^{E}$. To conclude that $w x \in W_{D}^{A}$, we need only show that for all $t \in E, w x \cdot x^{-1} t x>_{B} w x$ if $t x>_{B} x$, and $w x \cdot x^{-1} t x<_{B} w x$ if $t x<_{B} x$. Since $w x \cdot x^{-1} t x=w t x$, the former case follows immediately from Lemma 10.4. The latter case also follows from Lemma 10.4 , since $t x<_{B} x$ is equivalent to $t \cdot t x>_{B} t x$, which by the lemma implies that $w x=w \cdot t \cdot t x>_{B} w t x$.

Conversely, suppose $w \notin W^{E}$. Let $t \in E$ be such that $w t<_{B} w$. Now we show that if $t x>_{B} x$ then $w x \cdot x^{-1} t x<_{B} w x$, and if $t x<_{B} x$ then $w x \cdot x^{-1} t x>_{B} w x$. Again we apply Lemma 10.4 to both cases. In the former case, we have $w t \cdot t>_{B} w t$ and $t x>_{B} x$ which by Lemma 10.4 implies $w x \cdot x^{-1} t x=w t x<_{B} w t \cdot t \cdot x=w x$. In the latter case, we have $w t \cdot t>_{B} w t$ and $t \cdot t x>_{B} t x$, which by Lemma 10.4 implies

$$
w x \cdot x^{-1} t x=w t x=w t \cdot t \cdot t x>_{B} w t \cdot t x=w x .
$$

This leads us to conclude that $w x \notin W_{D}^{A}$, and hence that $C=W^{E} \cdot x=W_{D}^{A}$.

COROLlaRY 10.5. For $x_{1}, x_{2}, \ldots, x_{n} \in W$,

$$
\operatorname{Conv}\left(x_{1}, \ldots, x_{n}\right)=\left\{z \in W \mid \tilde{T}_{x_{1}} \cap \cdots \cap \tilde{T}_{x_{n}} \subseteq \tilde{T}_{z} \subseteq \tilde{T}_{x_{1}} \cup \cdots \cup \tilde{T}_{x_{n}}\right\} .
$$

ProOF. The right-hand side of the above equation is clearly equal to $W_{D}^{A}$, where $A=\tilde{T}_{x_{1}} \cap \cdots \cap \tilde{T}_{x_{n}}$ and $D=T-\left(\tilde{T}_{x_{1}} \cup \cdots \cup \tilde{T}_{x_{n}}\right)$. By Theorem 5.3, $W_{D}^{A}$ is convex and therefore contains the left-hand side. Also by Theorem 5.3, the left-hand side is equal to $W_{F}^{E}$ for some $E, F \subseteq T$. Since $x_{1}, x_{2}, \ldots, x_{n} \in W_{F}^{E}$, we have $E \subseteq \tilde{T}_{x_{i}}$ for all $i=1,2, \ldots, n$. Hence, $E \subseteq A$ and similarly, $F \subseteq D$. It follows that $W_{D}^{A} \subseteq W_{F}^{E}$, and hence that the two sets are equal.

ACKNOWLEDGEMENT. We want to thank a referee for suggestions which have led to improvements in $\S \S 4$ and 5.

\section{REFERENCES}

1. G. E. Andrews, The theory of partitions, Addison-Wesley, Reading, Mass., 1976.

2. G. E. Andrews and D. Foata, Congruences for the q-secant numbers, European J. Combinatorics 1 (1980), 283-287.

3. G. Birkhoff, Lattice theory, 3rd ed., Amer. Math. Soc., Providence, R.I., 1967.

4. A. Björner, Shellable and Cohen-Macaulay partially ordered sets, Trans. Amer. Math. Soc. 260 (1980), 159-183. 
5. __ Orderings of Coxeter groups, Contemp. Math., vol. 34, Amer. Math. Soc., Providence, R.I., 1984, pp. 175-195.

6. __ On matroids, groups and exchange languages, Matroid Theory and its Applications (L. Lovász and A. Recski, eds.), Colloq. Math. Soc. János Bolyai, vol. 40, North-Holland, Amsterdam, 1985, pp. 25-60.

7. _ The weak ordering of a Coxeter group (to appear).

8. A. Björner and M. Wachs, Bruhat order of Coxeter groups and shellability, Adv. in Math. 43 (1982), 87-100.

9. __ On lexicographically shellable posets, Trans. Amer. Math. Soc. 277 (1983), 323-341.

10. ___ Permutation statistics and linear extensions of posets (to appear).

11. _,$q$-hook length formulas for forests J. Combin. Theory Ser. A (to appear).

12. N. Bourbaki, Groupes et algèbres de Lie, Chaps. 4, 5, 6, Eléments de Mathématiques, Fasc. 34, Hermann, Paris, 1968.

13. V. V. Deodhar, Some characterizations of Bruhat ordering on a Coxeter group and determination of the relative Möbius function, Invent. Math. 39 (1977), 187-198.

14. R. Dipper and G. James, Representations of Hecke algebras of general linear groups, Proc. London Math. Soc. (3) 52 (1986), 20-52.

15. J. Fürlinger and J. Hof bauer, q-Catalan numbers, J. Combin. Theory Ser. A 40 (1985), 248264.

16. A. M. Garsia and J. Remmel, Shuffles of permutations and the Kronecker product, Graphs and Combinatorics 1 (1985), 217-263.

17. D. E. Knuth, Sorting and searching, The Art of Computer Programming, vol. 3, AddisonWesley, Reading, Mass., 1973.

18. A. Nijenhuis and H. Wilf, Combinatorial algorithms, 2nd ed., Academic Press, New York, 1978.

19. R. P. Stanley, Ordered structures and partitions, Mem. Amer. Math. Soc., no. 119, 1972.

20. __ Binomial posets, Möbius inversion, and permutation enumeration, J. Combin. Theory 20 (1976), 336-356.

21. _ Weyl groups, the hard Lefschetz theorem, and the Sperner property, SIAM J. Algebraic and Discrete Methods 1 (1981), 168-184.

22. J. Tits, Buildings of spherical type and finite BN-pairs, Lecture Notes in Math., vol. 386, Springer-Verlag, Berlin, and New York, 1974.

23. D. N. Verma, Möbius inversion for the Bruhat ordering on a Weyl group, Ann. Sci. École Norm. Sup. 4 (1971), 393-399.

Department of Mathematics, Massachusetts institute of TeChnOlOgy, CAMBRIDGE, MASSACHUSETTS 02139

Department of Mathematics, University of Miami, Coral Gables, Florida 33124 (Current address of M. L. Wachs)

Current address (Anders Björner): Department of Mathematics, Royal Institute of Technology, 10044 Stockholm, Sweden 